TITLE:

\title{
Ultrafast exciton transfers in DNA and its nonlinear optical spectroscopy
}

\section{$\operatorname{AUTHOR}(S)$ :}

Kim, Hyeon-Deuk; Tanimura, Yoshitaka; Cho, Minhaeng

\section{CITATION:}

Kim, Hyeon-Deuk ... [et al]. Ultrafast exciton transfers in DNA and its nonlinear optical spectroscopy. The Journal of Chemical Physics 2008, 128: 135102

ISSUE DATE:

2008-04

URL:

http://hdl.handle.net/2433/54282

\section{RIGHT:}

Copyright (2008) American Institute of Physics. This article may be downloaded for personal use only. Any other use requires prior permission of the author and the American Institute of Physics. 


\title{
Ultrafast exciton transfers in DNA and its nonlinear optical spectroscopy
}

\author{
Kim Hyeon-Deuk, ${ }^{1, a)}$ Yoshitaka Tanimura, ${ }^{1}$ and Minhaeng $\mathrm{Cho}^{2, \mathrm{~b})}$ \\ ${ }^{1}$ Department of Chemistry, Kyoto University, Kyoto 606-8502, Japan \\ ${ }^{2}$ Department of Chemistry and Center for Multidimensional Spectroscopy, Korea University, and \\ Multidimensional Spectroscopy Laboratory, Korea Basic Science Institute, Seoul 136-701, Korea
}

(Received 26 November 2007; accepted 14 February 2008; published online 2 April 2008)

\begin{abstract}
We have calculated the nonlinear response function of a DNA duplex helix including the contributions from the exciton population and coherence transfers by developing an appropriate exciton theory as well as by utilizing a projector operator technique. As a representative example of DNA double helices, the $B$-form $(\mathrm{dA})_{10^{-}}(\mathrm{dT})_{10}$ is considered in detail. The Green functions of the exciton population and coherence transfer processes were obtained by developing the DNA exciton Hamiltonian. This enables us to study the dynamic properties of the solvent relaxation and exciton transfers. The spectral density describing the DNA base-solvent interactions was obtained by adjusting the solvent reorganization energy to reproduce the absorption and steady-state fluorescence spectra. The time-dependent fluorescence shift of the model DNA system is found to be ultrafast and it is largely determined by the exciton population transfer processes. It is further shown that the nonlinear optical spectroscopic techniques such as photon echo peak shift and two-dimensional photon echo can provide important information on the exciton dynamics of the DNA double helix. We have found that the exciton-exciton coherence transfer plays critical roles in the peculiar energy transfer and ultrafast memory loss of the initially created excitonic state in the DNA duplex helix. (C) 2008 American Institute of Physics. [DOI: 10.1063/1.2894843]
\end{abstract}

\section{INTRODUCTION}

Distributions of photolesions caused by sunlight depend on the sequence of DNA around the hot spots, indicating a cooperativity between nucleoside excited states. An example that a DNA defect, which is 16 base pairs separated from the photoexcitable rhodium intercalator, can be healed is a good evidence on such a strong correlation between DNA base excited states. ${ }^{1}$ There have been some speculations about the nature of excited states of DNA duplex, i.e., whether they are localized on each monomer base or delocalized over many bases. Addressing this issue on the nature of photoexcited states is invaluable for understanding excitation migrations along the chain of DNA bases and for elucidating the mechanism of photoinitiated lesion processes.

The absorption spectra of monomeric purines and pyrimidines bases were extensively studied. ${ }^{2,3}$ Also, the electronic coupling strengths ${ }^{3}$ and their dynamical changes due to conformational fluctuations of a $B$-DNA have been studied recently. ${ }^{4-6}$ Particularly, the time-resolved fluorescence spectroscopy was used to study ultrafast relaxation processes of monomeric excited states and to estimate the decay time constants. ${ }^{7,8}$ However, while excited states of a single base show ultrafast transitions, excited states of a DNA duplex helix composed of strongly coupled bases are rather stable and their lifetime is longer than those of a single base. ${ }^{9}$

Theoretically, Bittner developed a lattice Fermion model to describe an electron/hole separation as well as their migrations along the DNA helix. ${ }^{10,11}$ The electron/hole cou-

\footnotetext{
${ }^{a}$ Electronic mail: kim@kuchem.kyoto-u.ac.jp.

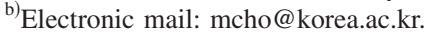

plings are caused by short-ranged Coulomb and exchange interactions. Not only the excited-state energy transfer processes but also the photoinitiated charge transfer processes were theoretically studied, which led to a conclusion that the excitation in a DNA duplex helix is delocalized over only a few bases. Essentially, the diagonal disorder considered in Ref. 11 is the main reason for such a relative localization of the excited state. In practice, it was assumed that the electronic coupling can be dealt with a point dipole-point dipole interaction, and its Hamiltonian was not diagonalized to ultimately obtain the exciton Hamiltonian. ${ }^{3}$ Furthermore, even though the off-diagonal disorder representing the structural fluctuations of the DNA bases in solution was taken into consideration, any couplings induced by phonon bath modes have not been included in the theoretical description of the DNA excited states. The latter is quite important because it is responsible for the time-dependent fluorescence shift and for properly describing decoherence processes, as will be shown in this paper.

Recently, it was found that the absorption spectra of duplex helices where the excited states are delocalized over bases only exhibit a slight shift with respect to the spectra of noninteracting single bases. ${ }^{4,12}$ The participation ratios of the $B$-DNA excited states, which are the measures of the numbers of bases involved in a given photoexcited state, were calculated and the results indicated that the excited states are delocalized over several bases. ${ }^{4-6,12}$ Markovitsi et al. for the first time carried out the subpicosecond time-resolved fluorescence spectroscopy of $(\mathrm{dA})_{20}(\mathrm{dT})_{20} \cdot{ }^{13}$ The absorption and ultrafast fluorescence spectral changes of other types of DNA helices such as poly $(\mathrm{dA})$ poly $(\mathrm{dT})$ and $(\mathrm{dAdT})_{10}(\mathrm{dAdT})_{10}$ were also investigated. ${ }^{14,15}$ In Ref. 16 , it was indicated that 
the subpicosecond decaying pattern of fluorescence anisotropy implies ultrafast excitation transfer taking place within the DNA helix. It was concluded that, since the excitation transfer rates are not proportional to the square of the electric couplings between bases, the transfer processes cannot be explained by the Förster theory. This is an important piece of information and indicates that the exciton representation can be an appropriate picture for the excited state of DNA double helix and that the excitation transfer processes should be described as exciton migrations on an excited-state manifold constructed by delocalized exciton states.

Furthermore, there are some other evidences on the delocalization of excited states. As discussed in Ref. 17, the delocalization length of DNA excited states is much larger than expected even at a few picoseconds after an excitation. Owing to the short base-base distances and the relatively rigid stacking structure of DNA helical duplexes, the excited states of bases in $(\mathrm{dA})_{n}(\mathrm{dT})_{n}$ are likely to be delocalized in comparison with those of a single-stranded sequence $(\mathrm{dA})_{n}$. Note that the $(\mathrm{dA})_{n}$ single strand is structurally highly flexible so that the interbase distance might have a broad distribution, which corresponds to relatively weak interbase electronic couplings. As a matter of fact, it was found that the time-dependent transient absorption spectral changes of the single strand $(\mathrm{dA})_{18}$ is fairly different from those of the $(\mathrm{dA})_{20}(\mathrm{dT})_{20}$ duplex. ${ }^{9}$ This experimental result is another strong evidence for exciton formation in a DNA duplex. The delocalization of excited energy created by sunlights generates dispersion of the ultraviolet energy which may lead to a photolesion and, as a result, DNA can avoid being damaged. There are also the quantum chemical and molecular dynamics simulations on the vibrational exciton dynamics of a variety of DNA duplex helices, which predicted the linear and two-dimensional (2D) vibrational spectra of polymorphic DNA helices. ${ }^{18}$

In the present study, we underscore the ultrafast exciton dynamics in a DNA duplex, which could be of importance for further understanding of the mechanism of UV lightinduced chemical reactions sometimes resulting in DNA mutations. Nevertheless, it has been experimentally difficult to carry out ultrafast nonlinear optical spectroscopic studies of DNA because they require femtosecond pulses with wavelengths shorter than $300 \mathrm{~nm}$. However, owing to the rapid development of ultrafast laser technology, it will be possible to experimentally investigate the ultrafast exciton transfer processes in DNA helices. Therefore, in the present paper, we will present a theory on exciton dynamics and migration within a DNA duplex helix and show that the nonlinear optical spectroscopic techniques such as photon echo peak shift ${ }^{19,20}$ (PEPS) and 2D electronic spectroscopy ${ }^{19,21}$ could provide critical information on the dynamics of photoexcited states such as ultrafast exciton transfers in a DNA duplex.

This paper is organized as follows. In Sec. II, we discuss the DNA excitation Hamiltonian in the base representation and recast it to the corresponding exciton representation. The theory of nonlinear optical spectroscopy and response function formalism will be discussed in Sec. III. In Sec. IV, the theoretical description on how to calculate the exciton population and coherence transfers will be presented in detail. The

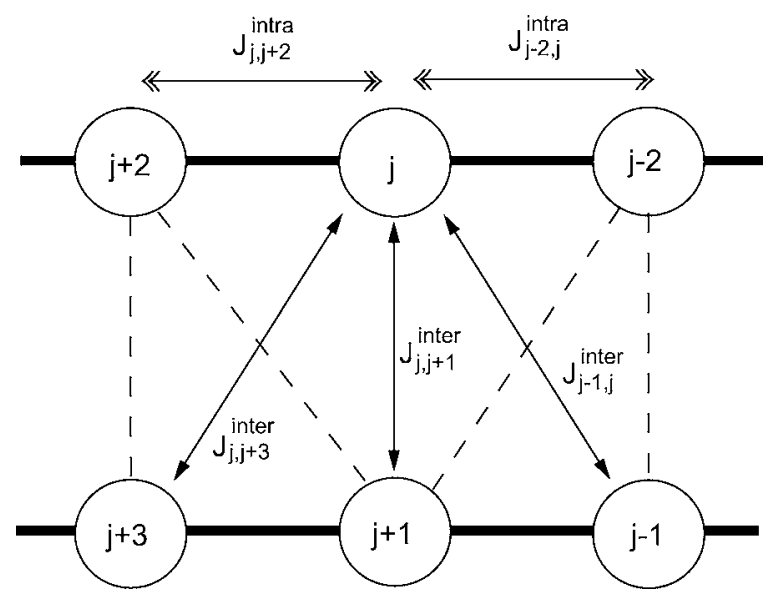

FIG. 1. A part of a two chain dimer which constitutes a DNA duplex helix. The base numbers appear on each base. The $j$ th base couplings between connected bases are shown as the normal and dual arrows for interchain and intrachain couplings, respectively. We only consider the nearest, next nearest, and diagonal couplings.

important material parameters for constructing the excitation Hamiltonian matrix of $B$-form DNA, e.g., $(\mathrm{dA})_{10^{-}}(\mathrm{dT})_{10}$, will be presented and discussed in Sec. V. The numerically calculated results of time-dependent fluorescence shift and various photon echo spectroscopic features will be provided in Secs. VI and VII, respectively. Our main results will be summarized in Sec. VIII.

\section{DNA EXCITON HAMILTONIAN}

Electronic states of a DNA duplex helix can be modeled as a strongly coupled multichromophore system consisting of $2 N$ two-level bases, where $N$ is the number of base pairs. Let us define the creation and annihilation operators $\hat{M}_{n}^{\dagger}$ and $\hat{M}_{n}$ that, respectively, creates and annihilates the excitation of $(n / 2)$ th base of a chain for even $n$ and that of $(n+1) / 2$ th base of the other chain for odd $n$ (see Fig. 1). We shall assume that each base interacts with neighboring bases only. The coupling constants can thus be classified as the intrastrand and interstrand couplings denoted as $J_{m n}^{\text {intra }}$ and $J_{m n}^{\text {inter }}$, respectively. Then, the DNA excitation Hamiltonian can be written as

$$
\begin{aligned}
\hat{H}= & \sum_{n} \Omega_{n} \hat{M}_{n}^{\dagger} \hat{M}_{n}+\sum_{m, n}^{|m-n|=2} J_{m n}^{\text {intra }} \hat{M}_{m}^{\dagger} \hat{M}_{n} \\
& +\sum_{m, n}^{|m-n|=1} J_{m n}^{\text {inter }} \hat{M}_{m}^{\dagger} \hat{M}_{n}+\sum_{m, n, k \geqslant 1}^{|m-n|=3, m+n=1+4 k} J_{m n}^{\text {inter }} \hat{M}_{m}^{\dagger} \hat{M}_{n} \\
& +\sum_{m, n} q_{m n}^{(c)} \hat{M}_{m}^{\dagger} \hat{M}_{n}+H_{\mathrm{ph}}\left(\left\{q_{j}\right\}\right)
\end{aligned}
$$

where $1 \leqslant n, m \leqslant 2 N$. The first term in Eq. (1) describes the base excitation energy and the next three terms represent electric couplings between base excitations. The fifth term describes the base-bath interaction and the last term is the phonon bath Hamiltonian given as 


$$
H_{\mathrm{ph}}\left(\left\{q_{j}\right\}\right)=\sum_{j}\left(\frac{p_{j}^{2}}{2 m_{j}}+\frac{m_{j} \omega_{j}^{2} q_{j}^{2}}{2}\right) .
$$

The coupling coefficient $q_{m n}^{(c)}$ is assumed to be linearly proportional to bath coordinates $\left\{q_{j}\right\}$,

$$
q_{m n}^{(c)}=\sum_{j} m_{j} \omega_{j}^{2} z_{j, m n} q_{j}
$$

Here, $z_{j, m n}$ is the coupling strength of the $j$ th phonon to the excitation operator $\hat{M}_{m}^{\dagger} \hat{M}_{n}$. If $m=n$ in Eq. (3), $q_{m m}^{(c)}$ modulates the excited-state energy of the $m$ th nucleoside. Otherwise, $q_{m n}^{(c)}$ induces fluctuation of the coupling constant between the $m$ th and $n$th nucleosides.

Although the DNA excitation Hamiltonian is useful in describing electronic properties of DNA duplex helices in the site representation, the above coupling constants are often large enough to make the excited states spatially delocalized over many nucleosides. Therefore, it is useful to recast the Hamiltonian [Eq. (1)] into the exciton representation by diagonalizing the first four terms. ${ }^{19,22}$ We note that, if the coupling constants are small compared to the base-bath interaction, the DNA excitation Hamiltonian [Eq. (1)] is acceptable and the excitation transfer rate from one base to another base can be calculated by the Förster theory and the rate becomes proportional to the square of $J_{m n}^{\text {intra }}$ or $J_{m n}^{\text {inter } 23}$ However, as mentioned in Sec. I, the recent experimental results report that excited states in DNA duplexes are spatially delocalized and that the transfer rate function does not directly depend on the square of electric couplings. Consequently, the exciton representation would be a more appropriate description for electronically excited states of DNA duplex helices.

In order to quantitatively describe conventional linear spectroscopy such as absorption and fluorescence, one needs information on the one-exciton states. Similarly, the nonlinear optical spectroscopy such as photon echo includes transitions from a one-exciton state to a two-exciton state too. Hereafter, $|0\rangle$ denotes the ground state, $|\mu\rangle$ the one-exciton state, and $|\bar{\mu}\rangle$ the two-exciton state (see Fig. 2). Then, the DNA excitation Hamiltonian [Eq. (1)] can be rearranged as

$$
\hat{H}_{e}=\hat{H}_{0}+\hat{H}_{1}
$$

where the nonperturbative part is

$$
\begin{aligned}
\hat{H}_{0}= & \sum_{\mu} \epsilon_{\mu} \hat{B}_{\mu}^{\dagger} \hat{B}_{\mu}+\sum_{\bar{\mu}} \epsilon_{\bar{\mu}} \hat{Y}_{\bar{\mu}}^{\dagger} \hat{Y}_{\bar{\mu}}+\sum_{\mu} q_{\mu \mu}^{(c)} \hat{B}_{\mu}^{\dagger} \hat{B}_{\mu} \\
& +\sum_{\bar{\mu}} q_{\bar{\mu} \bar{\mu}}^{(c)} \hat{Y}_{\bar{\mu}}^{\dagger} \hat{Y}_{\bar{\mu}}+H_{\mathrm{ph}}\left(\left\{q_{j}\right\}\right),
\end{aligned}
$$

and the perturbative part is given as

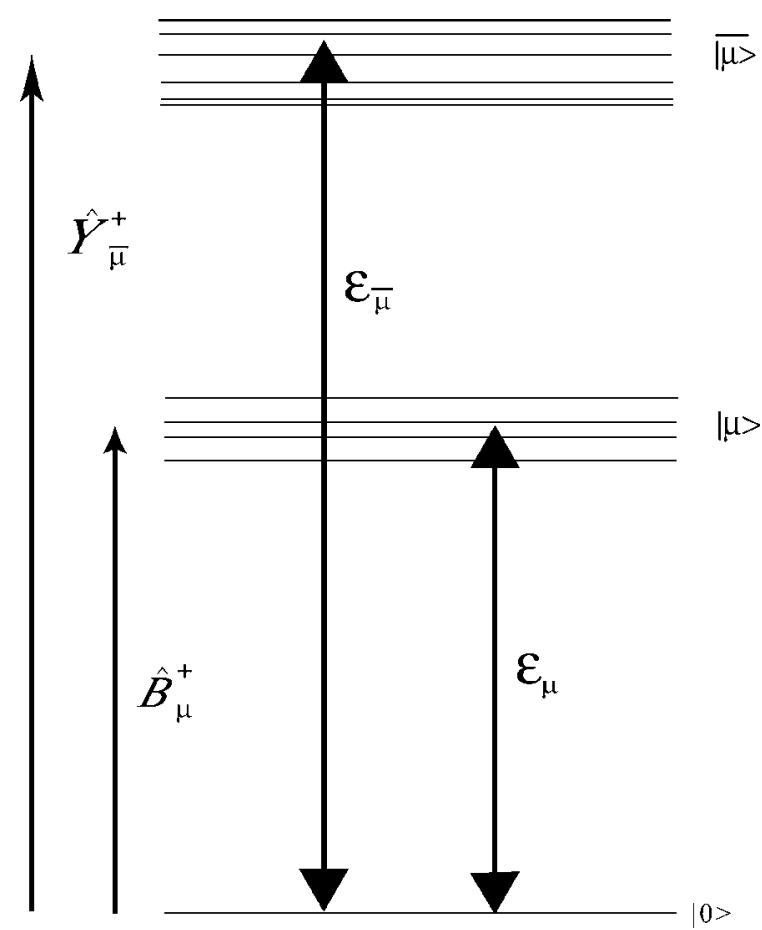

FIG. 2. Exciton level structure of the Hamiltonian [Eq. (5)]. $|0\rangle$ is the ground state, while $|\mu\rangle$ means one of the one-exciton levels consisting of $N$ states. $|\bar{\mu}\rangle$ denotes one of the $N(2 N-1)$ two-exciton states. $\epsilon_{\mu}$ and $\epsilon_{\bar{\mu}}$ are eigenenergies for the one- and two-exciton states, respectively. The exciton eigenenergies $\epsilon_{\mu}$ are numerically obtained by diagonalizing the first, second, third, and fourth terms of the DNA excitation Hamiltonian [Eq. (1)].

$$
\hat{H}_{1}=\sum_{\mu, \nu}^{\mu \neq \nu} q_{\mu \nu}^{(c)} \hat{B}_{\mu}^{\dagger} \hat{B}_{\nu}+\sum_{\bar{\mu}, \bar{\nu}}^{\bar{\mu} \neq \bar{\nu}} q_{\bar{\mu}}^{(c)} \hat{Y}_{\bar{\mu}}^{\dagger} \hat{Y}_{\bar{\nu}},
$$

with $1 \leqslant \mu, \nu \leqslant 2 N$ and $1 \leqslant \bar{\mu}, \bar{\nu} \leqslant N(2 N-1)$. We call $\hat{H}_{e}$ the DNA exciton Hamiltonian in order to distinguish it from the DNA excitation Hamiltonian [Eq. (1)]. $\hat{B}_{\mu}^{\dagger}$ and $\hat{B}_{\mu}$ are the exciton creation and annihilation operators for the oneexciton state $|\mu\rangle$, and the corresponding operators for the two-exciton state $|\bar{\mu}\rangle$ are expressed as $\hat{Y}_{\bar{\mu}}^{\dagger}$ and $\hat{Y}_{\bar{\mu}}$, respectively. $\epsilon_{\mu}$ and $\epsilon_{\bar{\mu}}$ are the eigenenergies of $|\mu\rangle$ and $|\bar{\mu}\rangle$, respectively. Due to the exciton-phonon couplings, the one- and two-exciton state energies undergo fluctuations in time, and they are expressed by $q_{\mu \mu}^{(c)}$ and $q_{\bar{\mu} \bar{\mu},}^{(c)}$, respectively. The offdiagonal exciton-phonon couplings, $q_{\mu \nu}^{(c)}$ and $q_{\bar{\mu} \bar{\nu}}^{(c)}$ can induce exciton transfers between different exciton population or coherent states. The detailed discussion and the explicit forms for $q_{\mu \nu}^{(c)}$ and $q_{\bar{\mu} \bar{\nu}}^{(c)}$ are given in Ref. 22.

\section{NONLINEAR RESPONSE FUNCTION AND SPECTROSCOPY}

Although the steady-state absorption and fluorescence spectra of DNA duplex helices can provide fundamental properties of their electronically excited states, the information extracted from such linear spectra is generally limited and highly averaged. In this regard, a number of nonlinear optical spectroscopic techniques with high time and frequency resolutions have been developed and applied to various molecular complexes. In this section, we will provide a 


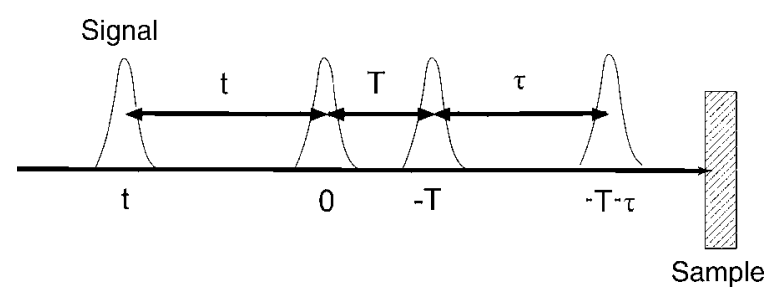

FIG. 3. A schematic description of the nonlinear spectroscopy. In the thirdorder nonlinear spectroscopy, three external pulse fields are operated to a sample. We set the time when the third pulse interacts with the sample zero, which makes the time when the probe signal is observed $t$.

brief outline of the nonlinear response function formalism in the nonlinear optical spectroscopy. The schematic configuration of optical laser pulses is drawn in Fig. 3, where the delay times $\tau$ and $T$ are experimentally controlled and the spectral interferometry detection of the dispersed signal is performed to obtain coherence evolution during time $t$ of a system.

In the third-order nonlinear spectroscopy, three external pulses are usually operated to interrogate an optical sample and the external Maxwell electric field is written as

$$
\begin{aligned}
E(\mathbf{r}, \tilde{t})= & E_{1} \delta\left(\tilde{t}-\left(t_{d}-t-T-\tau\right)\right)\left\{e^{i \mathbf{k}_{1} \cdot \mathbf{r}-i \omega_{1} \tilde{t}}+e^{-i \mathbf{k}_{1} \cdot \mathbf{r}+i \omega_{1} \tilde{t}}\right\} \\
& +E_{2} \delta\left(\tilde{t}-\left(t_{d}-t-T\right)\right)\left\{e^{i \mathbf{k}_{2} \cdot \mathbf{r}-i \omega_{2} \tilde{t}}+e^{-i \mathbf{k}_{2} \cdot \mathbf{r}+i \omega_{2} \tilde{t}}\right\} \\
& +E_{3} \delta\left(\tilde{t}-\left(t_{d}-t\right)\right)\left\{e^{i \mathbf{k}_{3} \cdot \mathbf{r}-i \omega_{3} \tilde{t}}+e^{-i \mathbf{k}_{3} \cdot \mathbf{r}+i \omega_{3} \tilde{t}}\right\}
\end{aligned}
$$

when each pulse envelopment is assumed to be a Dirac delta function. $t_{d}$ means a detection time. The $j$ th pulse amplitude, wave vector, and frequency are denoted as $E_{j}, \mathbf{k}_{j}$, and $\omega_{j}$, respectively. We will set the time when the third pulse interacts with the sample zero, i.e., $t_{d}=t$.

In order to obtain the third-order polarization in terms of the nonlinear response function, one can start with the quantum Liouville equation and use the time-ordered perturbation theory. ${ }^{24}$ The third-order polarization which is linearly proportional to the signal field is then found to be

$$
\begin{aligned}
P^{(3)}(\mathbf{r}, t, T, \tau)= & \int_{0}^{\infty} d t_{3} \int_{0}^{\infty} d t_{2} \int_{0}^{\infty} d t_{1} R^{(3)}\left(t_{3}, t_{2}, t_{1}\right) \\
& \times E\left(\mathbf{r}, t-t_{3}\right) E\left(\mathbf{r}-t_{3}-t_{2}\right) E\left(\mathbf{r}-t_{3}-t_{2}-t_{1}\right) .
\end{aligned}
$$

Although the above expression is general for all kinds of nonlinear optical polarization, we will focus on photon echo of which signal field's wave vector is $\mathbf{k}_{s}=-\mathbf{k}_{1}+\mathbf{k}_{2}+\mathbf{k}_{3}$. Substituting the external electric field [Eq. (7)] into Eq. (8) and performing the triple integrations, the observable third-order photon echo polarization is simply obtained as

$$
P^{(3)}(\mathbf{r}, t, T, \tau)=P_{\mathbf{k}_{s}}^{(3)}(t, T, \tau) e^{i\left(-\mathbf{k}_{1}+\mathbf{k}_{2}+\mathbf{k}_{3}\right) \cdot \mathbf{r}+i\left(\omega_{1}-\omega_{2}-\omega_{3}\right) t},
$$

where the third-order polarization component satisfying the phase-matching condition is given as

$P_{\mathbf{k}_{s}}^{(3)}(t, T, \tau)=R^{(3)}(t, T, \tau) E_{1} E_{2} E_{3} e^{-i \omega_{1}(t+T+\tau)+i \omega_{2}(t+T)+i \omega_{3} t}$.

As this expression shows, the signal field is essentially proportional to the nonlinear response function $R^{(3)}\left(t_{3}, t_{2}, t_{1}\right)$. Therefore, the remaining task is to theoretically calculate this nonlinear response function for DNA duplex helices.

The nonlinear response function is indispensable to calculate the nonlinear spectroscopic observables. Using the time-evolution propagator in the Liouville space, one can obtain the third-order response function as

$$
R^{(3)}\left(t_{3}, t_{2}, t_{1}\right)=i^{3} \operatorname{Tr}\left[\hat{d} \hat{G}\left(t_{3}\right) \hat{d}^{\times} \hat{G}\left(t_{2}\right) \hat{d}^{\times} \hat{G}\left(t_{1}\right) \hat{d}^{\times} \hat{\rho}_{00}\right],
$$

where the equilibrium density matrix operator in the ground state is

$$
\hat{\rho}_{00}\left(\left\{q_{j}\right\}\right)=|0\rangle \frac{\exp \left[-\beta H_{\mathrm{ph}}\left(\left\{q_{j}\right\}\right)\right]}{Z}\langle 0|,
$$

with $\beta=1 / k_{B} T_{B}$ and the normalization constant $Z ; k_{B}$ and $T_{B}$ are the Boltzmann constant and the temperature, respectively. ${ }^{24}$ The Liouville path propagator is defined as $\hat{G}(t) \equiv \exp [-i \hat{L} t]$. Here, $\hat{L} \hat{X}=\left[\hat{H}_{e}, \hat{X}\right]$ and $\hat{d}^{\times}$is the electric dipole hyperoperator, $\hat{d}^{\times} \hat{X} \equiv \hat{d} \hat{X}-\hat{X} \hat{d}$. The dipole operator in the exciton representation can be written in terms of the creation and annihilation operators of one- and two-exciton states as

$$
\hat{d}=\sum_{\mu} d_{\mu}\left(\hat{B}_{\mu}+\hat{B}_{\mu}^{\dagger}\right)+\sum_{\mu, \bar{\mu}} d_{\mu, \bar{\mu}}\left(\hat{Y}_{\bar{\mu}}^{\dagger} \hat{B}_{\mu}+\hat{B}_{\mu}^{\dagger} \hat{Y}_{\bar{\mu}}\right),
$$

where $d_{\mu}=\Sigma_{m} d_{m} \phi_{\mu}(m)$ and

$$
d_{\mu, \bar{\mu}}=\sum_{m=1}^{N-1} \sum_{n=m+1}^{N} \Psi_{\bar{\mu}}(m, n)\left\{\phi_{\mu}(n) d_{m}+\phi_{\mu}(m) d_{n}\right\} .
$$

Here, $\phi_{\mu}(m)$ and $\Psi_{\bar{\mu}}(m, n)$ represent the one- and twoexciton wave functions, respectively.

In order to calculate the third-order response function given in Eq. (11), we have to consider time evolutions of the diagonal and off-diagonal components of density operator such as $\rho_{00}\left(\left\{q_{j}\right\}\right), \quad \rho_{0 \mu}\left(\left\{q_{j}\right\}\right), \quad \rho_{0 \bar{\mu}}\left(\left\{q_{j}\right\}\right), \quad \rho_{\mu \nu}\left(\left\{q_{j}\right\}\right)$, and $\rho_{\mu \bar{\mu}}\left(\left\{q_{j}\right\}\right)$, which can be efficiently treated by the projection operator method. Now, the projection operator that extracts the diagonal component of $\rho=\rho\left(\left\{q_{j}\right\}\right)$ is defined as

$$
\mathrm{P}_{\mu} \rho \equiv \bar{\rho}_{\mu \mu}\left(\left\{q_{j}\right\}\right) \operatorname{Tr}_{\left\{q_{j}\right\}}\left\{\rho_{\mu \mu}\right]=\hat{\rho}_{\mu \mu}
$$

where the equilibrium density matrix of the $\mu$ th exciton state is

$$
\bar{\rho}_{\mu \mu}\left(\left\{q_{j}\right\}\right)=\frac{\exp \left[-\beta H_{\mu \mu}\left(\left\{q_{j}\right\}\right)\right]}{Z},
$$


and $H_{\mu \mu}\left(\left\{q_{j}\right\}\right)$ is the nuclear Hamiltonian of the $\mu$ th exciton state. These definitions can also be seen in Refs. 23 and 25. Owing to the nonperturbative diagonal exciton-phonon couplings in Eq. (5), $H_{\mu \mu}\left(\left\{q_{j}\right\}\right)-\epsilon_{\mu}$ differs from the simple phonon bath $H_{\mathrm{ph}}\left(\left\{q_{j}\right\}\right)$ in Eq. (12). This means that phonon bath with which a particular exciton interacts is not degenerate. Thus, each exciton-state energy fluctuates in time differently and the time evolution of the projected density matrix is determined by different heat baths from one another. This is one of the essential differences between the Redfield theory and the present exciton transfer theory. ${ }^{23}$ From the definition of the projection operator [Eq. (15)], the ground-state population component is $\mathrm{P}_{0} \rho \equiv \bar{\rho}_{00} \operatorname{Tr}_{\left\{g_{j}\right\}}\left[\rho_{00}\right]$. The total projection operator is therefore given as $\mathrm{P}=\mathrm{P}_{0}+\Sigma_{\mu} \mathrm{P}_{\mu}$, while the complementary operator leads to $\mathrm{Q} \equiv 1-\mathrm{P}$.

Substitution of the expanded form of the Liouville space time-evolution operator, $\hat{G}\left(t_{2}\right)=\hat{G}_{\mathrm{PP}}\left(t_{2}\right)+\hat{G}_{\mathrm{PQ}}\left(t_{2}\right)+\hat{G}_{\mathrm{QP}}\left(t_{2}\right)$ $+\hat{G}_{\mathrm{QQ}}\left(t_{2}\right)$, into Eq. (11) leads to

$$
\begin{aligned}
R^{(3)}\left(t_{3}, t_{2}, t_{1}\right)= & i^{3} \operatorname{Tr}\left[\hat{d} \hat{G}_{\mathrm{QQ}}\left(t_{3}\right) \hat{d}^{\times} \hat{G}_{\mathrm{PP}}\left(t_{2}\right) \hat{d}^{\times} \hat{G}_{\mathrm{QQ}}\left(t_{1}\right) \hat{d}^{\times} \hat{\rho}_{00}\right] \\
& +i^{3} \operatorname{Tr}\left[\hat{d} \hat{G}_{\mathrm{QQ}}\left(t_{3}\right) \hat{d}^{\times} \hat{G}_{\mathrm{PQ}}\left(t_{2}\right) \hat{d}^{\times} \hat{G}_{\mathrm{QQ}}\left(t_{1}\right) \hat{d}^{\times} \hat{\rho}_{00}\right] \\
& +i^{3} \operatorname{Tr}\left[\hat{d} \hat{G}_{\mathrm{QQ}}\left(t_{3}\right) \hat{d}^{\times} \hat{G}_{\mathrm{QP}}\left(t_{2}\right) \hat{d}^{\times} \hat{G}_{\mathrm{QQ}}\left(t_{1}\right) \hat{d}^{\times} \hat{\rho}_{00}\right] \\
& +i^{3} \operatorname{Tr}\left[\hat{d} \hat{G}_{\mathrm{QQ}}\left(t_{3}\right) \hat{d}^{\times} \hat{G}_{\mathrm{QQ}}\left(t_{2}\right) \hat{d}^{\times} \hat{G}_{\mathrm{QQ}}\left(t_{1}\right) \hat{d}^{\times} \hat{\rho}_{00}\right],
\end{aligned}
$$

where $\hat{G}_{\mathrm{AB}}(t) \equiv \mathrm{A} \hat{G}(t) \mathrm{B}$. It should be noted that, during $t_{1}$ and $t_{3}$, the density matrix is always in one of the electronic coherent states between the ground and excitonic states such as $\hat{\rho}_{0 \mu}, \hat{\rho}_{0 \bar{\mu}}, \hat{\rho}_{\mu \nu}$, and $\hat{\rho}_{\mu \bar{\nu}}$. These states belong not to the $\mathrm{P}$-projected space but to the $\mathrm{Q}$-projected space since the former includes only diagonal population states. Therefore, the time evolutions of the system during $t_{1}$ and $t_{3}$ are determined by only $\hat{G}_{\mathrm{QQ}}(t)$.

The entire Liouville operator $\hat{L}$ is now divided into the perturbation and nonperturbation parts, $\hat{L}=\hat{L}_{0}+\hat{L}_{1}$. As shown in Refs. 19,25 , and 26 , to the lowest order of $\hat{L}_{1}$, we have

$$
\begin{aligned}
R^{(3)}\left(t_{3}, t_{2}, t_{1}\right) & \\
= & i^{3} \operatorname{Tr}\left[\hat{d} \hat{G}_{\mathrm{QQ}}\left(t_{3}\right) \hat{d}^{\times} \hat{G}_{\mathrm{PP}}\left(t_{2}\right) \hat{d}^{\times} \hat{G}_{\mathrm{QQ}}\left(t_{1}\right) \hat{d}^{\times} \hat{\rho}_{00}\right] \\
& +i^{3} \operatorname{Tr}\left[\hat{d} \hat{G}_{\mathrm{QQ}}\left(t_{3}\right) \hat{d}^{\times} \hat{G}_{\mathrm{QQ}}\left(t_{2}\right) \hat{d}^{\times} \hat{G}_{\mathrm{QQ}}\left(t_{1}\right) \hat{d}^{\times} \hat{\rho}_{00}\right] .
\end{aligned}
$$

This truncation does not mean the simple secular approximation in the Redfield theory, as will be discussed in Sec. VIII. The first term in Eq. (18) leads to the contribution including the exciton population transfer (EPT), $R_{\mathrm{EPT}}\left(t_{3}, t_{2}, t_{1}\right)$. As shown in Ref. 19, the second term in Eq. (18) includes both the zeroth-order term $R^{(0)}\left(t_{3}, t_{2}, t_{1}\right)$ and the contribution $R_{\text {EECT }}\left(t_{3}, t_{2}, t_{1}\right)$ from the exciton-exciton coherence transfer (EECT). The contributions including the EPT and EECT, $R_{\mathrm{EPT}}\left(t_{3}, t_{2}, t_{1}\right)$ and $R_{\mathrm{EECT}}\left(t_{3}, t_{2}, t_{1}\right)$, will be discussed in the next section.

The zeroth-order solution $R^{(0)}\left(t_{3}, t_{2}, t_{1}\right)$ includes none of the exciton transfers, and its explicit form for photon echo response function was given in Eqs. (D1)-(D4) of Ref. 25 as

$$
R^{(0)}\left(t_{3}, t_{2}, t_{1}\right)=R_{\mathrm{I}}\left(t_{3}, t_{2}, t_{1}\right)+R_{\mathrm{II}}\left(t_{3}, t_{2}, t_{1}\right)+R_{\mathrm{III}}\left(t_{3}, t_{2}, t_{1}\right),
$$

with

$$
\begin{aligned}
R_{\mathrm{I}}\left(t_{3}, t_{2}, t_{1}\right)= & -i \sum_{\mu, \nu} d_{\mu}^{2} d_{\nu}^{2} \exp \left[-i \epsilon_{\mu}\left(t_{3}+t_{2}\right)+i \epsilon_{\nu}\left(t_{2}+t_{1}\right)\right. \\
& \left.-f_{\mu \nu}^{(1)}\left(0, t_{2}+t_{1}, t_{3}+t_{2}+t_{1}, t_{1}\right)\right] \\
R_{\mathrm{II}}\left(t_{3}, t_{2}, t_{1}\right)= & -i \sum_{\mu, \nu} d_{\mu}^{2} d_{\nu}^{2} \exp \left[-i \epsilon_{\mu} t_{3}+i \epsilon_{\nu} t_{1}\right. \\
& \left.-f_{\mu \nu}^{(1)}\left(0, t_{1}, t_{3}+t_{2}+t_{1}, t_{2}+t_{1}\right)\right]
\end{aligned}
$$

and

$$
\begin{aligned}
R_{\mathrm{III}}\left(t_{3}, t_{2}, t_{1}\right)= & i \sum_{\mu, \nu, \bar{\mu}} d_{\mu, \bar{\mu}} d_{\nu, \bar{\mu}} d_{\mu} d_{\nu} \\
& \times \exp \left[i \epsilon_{\mu}\left(t_{3}+t_{2}+t_{1}\right)-i \epsilon_{\nu} t_{2}-i \epsilon_{\bar{\mu}} t_{3}\right. \\
& \left.-f_{\mu \nu, \bar{\mu}}^{(2) *}\left(t_{1}, t_{2}+t_{1}, t_{3}+t_{2}+t_{1}, 0\right)\right] .
\end{aligned}
$$

Here, the auxiliary functions in Eqs. (20)-(22) are defined by

$$
\begin{aligned}
f_{\mu \nu}^{(1)}\left(\tau_{4}, \tau_{3}, \tau_{2}, \tau_{1}\right)= & \exp \left[g_{\nu \nu, \nu \nu}\left(\tau_{4}-\tau_{3}\right)-g_{\nu \nu, \mu \mu}\left(\tau_{4}-\tau_{2}\right)\right. \\
& +g_{\nu \nu, \mu \mu}\left(\tau_{4}-\tau_{1}\right)+g_{\nu \nu, \mu \mu}\left(\tau_{3}-\tau_{2}\right) \\
& \left.-g_{\nu \nu, \mu \mu}\left(\tau_{3}-\tau_{1}\right)+g_{\mu \mu, \mu \mu}\left(\tau_{2}-\tau_{1}\right)\right]
\end{aligned}
$$

$$
\begin{aligned}
f_{\mu \nu, \bar{\mu}}^{(2)}\left(\tau_{4}, \tau_{3}, \tau_{2}, \tau_{1}\right)= & \exp \left[g_{\nu \nu, \nu \nu}\left(\tau_{4}-\tau_{3}\right)-g_{\nu \nu, \bar{\mu} \bar{\mu}}\left(\tau_{4}-\tau_{3}\right)+g_{\nu \nu, \bar{\mu} \bar{\mu}}\left(\tau_{4}-\tau_{2}\right)-g_{\nu \nu, \mu \mu}\left(\tau_{4}-\tau_{2}\right)+g_{\nu \nu, \mu \mu}\left(\tau_{4}-\tau_{1}\right)-g_{\nu \nu, \bar{\mu} \bar{\mu}}\left(\tau_{3}-\tau_{2}\right)\right. \\
& +g_{\nu \nu, \mu \mu}\left(\tau_{3}-\tau_{2}\right)-g_{\nu \nu, \mu \mu}\left(\tau_{3}-\tau_{1}\right)+g_{\bar{\mu} \bar{\mu}, \bar{\mu} \bar{\mu}}\left(\tau_{3}-\tau_{2}\right)-g_{\bar{\mu} \bar{\mu}, \mu \mu}\left(\tau_{3}-\tau_{2}\right)+g_{\bar{\mu} \bar{\mu}, \mu \mu}\left(\tau_{3}-\tau_{1}\right)-g_{\bar{\mu} \bar{\mu}, \mu \mu}\left(\tau_{2}-\tau_{1}\right) \\
& \left.+g_{\mu \mu, \mu \mu}\left(\tau_{2}-\tau_{1}\right)\right] .
\end{aligned}
$$


The exciton line-broadening function $g_{\alpha \gamma, \alpha^{\prime} \gamma^{\prime}}(t)$ can be written in terms of a spectral density describing the spectral distribution of exciton-phonon interactions,

$$
\begin{aligned}
g_{\alpha \gamma, \alpha^{\prime} \gamma^{\prime}}(t) \equiv \int_{0}^{t} d \tau^{\prime} \int_{0}^{\tau^{\prime}} d \tau^{\prime \prime}\left\langle q_{\alpha \gamma}^{(c)}\left(\tau^{\prime \prime}\right) q_{\alpha^{\prime} \gamma^{\prime}}^{(c)}(0)\right\rangle, \\
=\int_{-\infty}^{\infty} \frac{d \omega}{2 \pi} \frac{1-\cos \omega t}{\omega^{2}} \operatorname{coth} \frac{\beta \hbar \omega}{2} C_{\alpha \gamma, \alpha^{\prime} \gamma^{\prime}}(\omega) \\
+i \int_{-\infty}^{\infty} \frac{d \omega}{2 \pi} \frac{\sin \omega t-\omega t}{\omega^{2}} C_{\alpha \gamma, \alpha^{\prime} \gamma^{\prime}}(\omega),
\end{aligned}
$$

where the exciton spectral density is defined as

$$
C_{\alpha \gamma, \alpha^{\prime} \gamma^{\prime}}(\omega) \equiv \frac{1}{2} \int_{-\infty}^{\infty} d t \exp [i \omega t]\left\langle\left[q_{\alpha \gamma}^{(c)}(t), q_{\alpha^{\prime} \gamma^{\prime}}^{(c)}(0)\right]\right\rangle .
$$

The exciton homogeneous parameter is obtained from

$$
\kappa_{\alpha \gamma, \alpha^{\prime} \gamma^{\prime}} \equiv-\lim _{\tau \rightarrow \infty} \operatorname{Im}\left[\frac{d g_{\alpha \gamma, \alpha^{\prime} \gamma^{\prime}}(\tau)}{d \tau}\right] .
$$

\section{POPULATION AND COHERENCE TRANSFERS OF EXCITONS}

In this section, we will present a brief discussion on how to take into account the contributions of EPT and EECT to the nonlinear response function, that is, how to calculate $R_{\mathrm{EPT}}\left(t_{3}, t_{2}, t_{1}\right)$ and $R_{\mathrm{EECT}}\left(t_{3}, t_{2}, t_{1}\right)$. The EPT process and its effect on the nonlinear optical signals of the light-harvesting complexes were already discussed. ${ }^{19,22,25}$ The corresponding EPT response function, the first term of Eq. (18), in the doorway-window picture is given as

$$
\begin{aligned}
& i^{3} \sum_{\mu, \nu} \operatorname{Tr}\left[\hat{d} \hat{G}_{\mathrm{QQ}}\left(t_{3}\right) \hat{d}^{\times} \mathrm{P}_{\mu} \hat{G}\left(t_{2}\right) \mathrm{P}_{\nu} \hat{d}^{\times} \hat{G}_{\mathrm{QQ}}\left(t_{1}\right) \hat{d}^{\times} \hat{\rho}_{00}\right]= \\
& -i \sum_{\mu, \nu} \operatorname{Tr}\left[\hat{d} \hat{G}_{\mathrm{QQ}}\left(t_{3}\right) \hat{d}^{\times} \hat{G}_{\mu \mu, \nu \nu}\left(t_{2}\right) \hat{d}^{\times} \hat{G}_{\mathrm{QQ}}\left(t_{1}\right) \hat{d}^{\times} \hat{\rho}_{00}\right], \\
& \simeq-i \sum_{\mu, \nu} W_{\mu \mu}\left(t_{3}\right) G_{\mu \mu, \nu \nu}\left(t_{2}\right) D_{\nu \nu}\left(t_{1}\right)+i \sum_{\mu} W_{\mu \mu}\left(t_{3}\right) D_{\mu \mu}\left(t_{1}\right), \\
& \equiv R_{\mathrm{EPT}}\left(t_{3}, t_{2}, t_{1}\right)-R_{\mathrm{EPT}}\left(t_{3}, 0, t_{1}\right) .
\end{aligned}
$$

The last term corresponds to the doorway-window term, $i \Sigma_{\mu} W_{\mu \mu}\left(t_{3}\right) D_{\mu \mu}\left(t_{1}\right)$ at $t_{2}=0$, which has to be added to the EPT contribution since there is no EPT contribution at $t_{2}$ $=0$. Here, the doorway and window functions that describe the time evolution of the system's electronic coherence during $t_{1}$ and $t_{3}$ were found to be

$$
D_{\nu \nu}\left(t_{1}\right) \equiv \operatorname{Tr}\left[\hat{d}^{\times} \hat{G}_{\mathrm{QQ}}\left(t_{1}\right) \hat{d}^{\times} \hat{\rho}_{00}\right]
$$

and

$$
W_{\mu \mu}\left(t_{3}\right) \equiv \operatorname{Tr}\left[\hat{d} \hat{G}_{\mathrm{QQ}}\left(t_{3}\right) \hat{d}^{\times} \hat{\rho}_{\mu \mu}\right],
$$

respectively. Their explicit expressions for photon echo spectroscopy were obtained as

$$
D_{\nu \nu}\left(t_{1}\right)=d_{\nu}^{2} \exp \left[-i \epsilon_{\nu} t_{1}-g_{\nu \nu, \nu \nu}\left(t_{1}\right)\right]
$$

and

$$
\begin{aligned}
W_{\mu \mu}\left(t_{3}\right)= & d_{\mu}^{2} \exp \left[i \epsilon_{\mu} t_{3}-g_{\mu \mu, \mu \mu}\left(t_{3}\right)-2 i \kappa_{\mu \mu} t_{3}\right] \\
& -\sum_{\bar{\mu}} d_{\mu, \bar{\mu}}^{2} \exp \left[i\left(\epsilon_{\bar{\mu}}-\epsilon_{\mu}\right) t_{3}-g_{\mu \mu, \mu \mu}^{*}\left(t_{3}\right)\right. \\
& \left.-g_{\bar{\mu} \bar{\mu}, \bar{\mu} \bar{\mu}}^{*}\left(t_{3}\right)+2 g_{\mu \mu, \bar{\mu} \bar{\mu}}^{*}\left(t_{3}\right)-2 i\left(\kappa_{\mu \bar{\mu}}-\kappa_{\mu \mu}\right) t_{3}\right] .
\end{aligned}
$$

The Green function describing ETP from a population state $\nu \nu$ to another population state $\mu \mu$ is defined as

$$
\hat{G}_{\mu \mu, \nu \nu}\left(t_{2}\right) \equiv \mathrm{P}_{\mu} \hat{G}\left(t_{2}\right) \mathrm{P}_{\nu} .
$$

This is a conditional probability of finding the population state $\mu \mu$ at time $t_{2}$ when the initial population state at $t_{2}=0$ was $\nu \nu$. The traced $\hat{G}_{\mu \mu, \nu \nu}\left(t_{2}\right)$ is defined as follows;

$$
G_{\mu \mu, \nu \nu}\left(t_{2}\right) \equiv \operatorname{Tr}\left[\mathrm{P}_{\mu} \hat{G}\left(t_{2}\right) \mathrm{P}_{\nu} \hat{\rho}_{\nu \nu}\right] .
$$

The time-evolution equation for $G_{\mu \mu, \nu \nu}(t)$ was derived and discussed in Ref. 25, and it is rewritten here for the sake of completeness,

$$
\begin{aligned}
\frac{\partial G_{\mu \mu, \nu \nu}(t)}{\partial t}= & \sum_{\alpha} \int_{0}^{t} d \tau\left\{K_{\mu \mu, \alpha \alpha}(t-\tau) G_{\alpha \alpha, \nu \nu}(\tau)\right. \\
& \left.-K_{\alpha \alpha, \mu \mu}(t-\tau) G_{\mu \mu, \nu \nu}(\tau)\right\} .
\end{aligned}
$$

The EPT rate kernel function is

$$
\begin{aligned}
K_{\mu \mu, \alpha \alpha}(t) & \equiv-\operatorname{Tr}\left[\mathrm{P}_{\mu} \hat{L}_{1} \hat{G}_{\mathrm{QQ}}(t) \hat{L}_{1} \hat{\rho}_{\alpha \alpha}\right] \\
& =K_{\mu \mu, \alpha \alpha}^{L}(t)+K_{\mu \mu, \alpha \alpha}^{L}(-t) .
\end{aligned}
$$

According to the theory of Zhang et al., the explicit form of $K_{\mu \mu, \nu \nu}^{L}(t)$ is

$$
\begin{aligned}
K_{\mu \mu, \nu \nu}^{L}(t)= & \exp \left[-i\left(\epsilon_{\mu}-\epsilon_{\nu}\right) t-g_{\mu \mu, \mu \mu}(t)-g_{\nu \nu, \nu \nu}(t)\right. \\
& \left.+g_{\nu \nu, \mu \mu}(t)+g_{\mu \mu, \nu \nu}(t)-2 i\left(\kappa_{\nu \nu, \nu \nu}-\kappa_{\mu \mu, \nu \nu}\right) t\right] \\
& \times\left\{\ddot{g}_{\mu \nu, \nu \mu}(t)-\left[\dot{g}_{\nu \mu, \nu \nu}(t)-\dot{g}_{\nu \mu, \mu \mu}(t)\right.\right. \\
& \left.\left.+2 i \kappa_{\nu \mu, \nu \nu}\right]\left[\dot{g}_{\nu \nu, \mu \nu}(t)-\dot{g}_{\mu \mu, \mu \nu}(t)+2 i \kappa_{\mu \nu, \nu \nu}\right]\right\} .
\end{aligned}
$$

Here, the exciton line-broadening function $g_{\alpha \gamma, \alpha^{\prime} \gamma^{\prime}}(t)$ was given in Eq. (26). The explicit form of the exciton spectral density defined in Eq. (27) is related to the spectral density of base-phonon interactions as

$$
C_{\mu \nu, \mu^{\prime} \nu^{\prime}}(\omega)=\sum_{m, n, k, l} \phi_{\mu}(m) \phi_{\nu}^{*}(n) \phi_{u^{\prime}}(k) \phi_{\nu^{\prime}}^{*}(l) C_{m n, k l}(\omega),
$$

where the base-phonon spectral density is defined as 


$$
C_{m n, k l}(\omega) \equiv \frac{1}{2} \int_{-\infty}^{\infty} d t \exp [i \omega t] \tilde{C}_{m n, k l}(t)
$$

with $\widetilde{C}_{m n, k l}(t)=\left\langle\left[q_{m n}^{(c)}(t), q_{k l}^{(c)}(0)\right]\right\rangle$. The statistical average $\langle\cdots\rangle$ is performed over $\exp \left[-\beta H_{\mathrm{ph}}\left(\left\{q_{j}\right\}\right)\right] / Z$. It is assumed that the collective phonon variables acting on different bases are statistically uncorrelated and have the same spectral density, that is,

$$
C_{m n, k l}(\omega)=\delta_{m n} \delta_{k l} \delta_{m k} C(\omega) .
$$

In the present work, we use the spectral density of overdamped Brownian oscillator,

$$
C(\omega)=2 \kappa \frac{\omega \tau_{B}}{\omega^{2} \tau_{B}^{2}+1},
$$

with the relaxation time $\tau_{B}$ of an exponentially decaying noise correlation function. ${ }^{27}$ The homogeneous parameter $\kappa$ determines the absolute magnitude of base-phonon coupling strength and is identical to the solvent reorganization energy when a single base molecule undergoes an excitation in a DNA helix. In order to calculate the nonlinear response function with and without the exciton transfers in the exciton representation, it is also necessary to evaluate the exciton homogeneous parameter defined in Eq. (28) which is related to $\kappa$ as

$$
\kappa_{\mu \nu, \mu^{\prime} \nu^{\prime}}=\sum_{m, n, k, l} \phi_{\mu}(m) \phi_{\nu}^{*}(n) \phi_{\mu^{\prime}}(k) \phi_{\nu^{\prime}}^{*}(l) \kappa .
$$

Equation (38) with the rate kernel function Eqs. (39) and (40) describes EPT between different exciton population states. However, as discussed in Ref. 19, there is another possible exciton transfer, EECT, which includes off-diagonal components of the density matrix, i.e., exciton coherence. The EECT processes had been supposed to be much faster than the EPT processes so that they have not been considered in detail. In fact, only recently, some experimental evidences of coherence transfer in electronic and vibrational multichromophore systems have been reported. ${ }^{21,28}$ For instance, the advancement of ultrafast laser technology has allowed to observe time-resolved coherence transfer processes of the lightharvesting protein complex. It has been revealed that the coherence transfers play an important role in describing 2D spectroscopy of coupled multichromophore systems, in general.

As briefly mentioned in the previous section, the second term in Eq. (18) includes the EECT contribution to the nonlinear response function. It can be rearranged as

$$
\begin{aligned}
i^{3} \operatorname{Tr}\left[\hat{d} \hat{G}_{\mathrm{QQ}}\left(t_{3}\right) \hat{d}^{\times} \hat{G}_{\mathrm{QQ}}\left(t_{2}\right) \hat{d}^{\times} \hat{G}_{\mathrm{QQ}}\left(t_{1}\right) \hat{d}^{\times} \hat{\rho}_{00}\right] & =i^{3} \sum_{\mu \nu, \mu^{\prime} \nu^{\prime}} \operatorname{Tr}\left[\hat{d} \hat{G}_{\mathrm{QQ}}\left(t_{3}\right) \hat{d}^{\times} \mathrm{Q}_{\mu \nu} \hat{G}\left(t_{2}\right) \mathrm{Q}_{\mu^{\prime} \nu^{\prime}} \hat{d}^{\times} \hat{G}_{\mathrm{QQ}}\left(t_{1}\right) \hat{d}^{\times} \hat{\rho}_{00}\right], \\
& =-i \sum_{\mu \nu, \mu^{\prime} \nu^{\prime}} \operatorname{Tr}\left[\hat{d} \hat{G}_{\mathrm{QQ}}\left(t_{3}\right) \hat{d}^{\times} \hat{G}_{\mu \nu, \mu^{\prime} \nu^{\prime}}\left(t_{2}\right) \hat{d}^{\times} \hat{G}_{\mathrm{QQ}}\left(t_{1}\right) \hat{d}^{\times} \hat{\rho}_{00}\right], \\
& \simeq R^{(0)}\left(t_{3}, t_{2}, t_{1}\right)-i \sum_{\mu \nu, \mu^{\prime} \nu^{\prime}} W_{\mu \nu}\left(t_{3}\right) G_{\mu \nu, \mu^{\prime} \nu^{\prime}}\left(t_{2}\right) D_{\mu^{\prime} \nu^{\prime}}\left(t_{1}\right)+i \sum_{\mu \nu} W_{\mu \nu}\left(t_{3}\right) D_{\mu \nu}\left(t_{1}\right), \\
& \equiv R^{(0)}\left(t_{3}, t_{2}, t_{1}\right)+R_{\mathrm{EECT}}\left(t_{3}, t_{2}, t_{1}\right)-R_{\mathrm{EECT}}\left(t_{3}, 0, t_{1}\right)
\end{aligned}
$$

The second term of Eq. (48) in the doorway-window picture describes the EECT process and is denoted as $R_{\mathrm{EECT}}\left(t_{3}, t_{2}, t_{1}\right)$. The third term, $-R_{\mathrm{EECT}}\left(t_{3}, 0, t_{1}\right)$, is required because there should be no EECT contribution at $t_{2}=0 .{ }^{29}$ Such term is also needed in the case of EPT, that is, the second term in Eq. (31). Note that EECT processes during $t_{1}$ and $t_{3}$ can be ignored since there is no such system-bath interactions inducing transitions between the ground and a one-exciton state or between one- and two-exciton states. ${ }^{19}$ In Eq. (48), the doorway and window functions are

$$
D_{\mu^{\prime} \nu^{\prime}}\left(t_{1}\right) \equiv \operatorname{Tr}\left[\left(\mu^{\prime} \nu^{\prime}\right)^{\dagger} \hat{d}^{\times} \hat{G}_{\mathrm{QQ}}\left(t_{1}\right) \hat{d}^{\times} \hat{\rho}_{00}\right]
$$

and

$$
W_{\mu \nu}\left(t_{3}\right) \equiv \operatorname{Tr}\left[\hat{d} \hat{G}_{\mathrm{QQ}}\left(t_{3}\right) \hat{d}^{\times} \hat{\rho}_{\mu \nu}\right],
$$

respectively. As will be shown later, $\left(\mu^{\prime} \nu^{\prime}\right)^{\dagger}$ in the doorway function is needed to produce $\hat{\rho}_{\mu^{\prime} \nu^{\prime}}$ in the EECT Green function $G_{\mu \nu, \mu^{\prime} \nu^{\prime}}\left(t_{2}\right)$. Their explicit expressions for photon echo are given in Ref. 19 as

$$
D_{\mu^{\prime} \nu^{\prime}}\left(t_{1}\right)=d_{\mu^{\prime}} d_{\nu^{\prime}} \exp \left[-i \epsilon_{\mu^{\prime}} t_{1}-g_{\mu^{\prime} \mu^{\prime}, \mu^{\prime} \mu^{\prime}}\left(t_{1}\right)\right]
$$

and

$$
\begin{aligned}
W_{\mu \nu}\left(t_{3}\right)= & d_{\mu} d_{\nu} \exp \left[i \epsilon_{\mu} t_{3}-g_{\mu \mu, \mu \mu}\left(-t_{3}\right)\right] \\
& -\sum_{\bar{\mu}} d_{\mu, \bar{\mu}} d_{\nu, \bar{\mu}} \exp \left[i\left(\epsilon_{\bar{\mu}}-\epsilon_{\mu}\right) t_{3}-g_{\mu \mu, \mu \mu}\left(t_{3}\right)\right. \\
& \left.-g_{\bar{\mu} \bar{\mu}, \bar{\mu} \bar{\mu}}^{*}\left(t_{3}\right)+g_{\bar{\mu} \bar{\mu}, \mu \mu}\left(t_{3}\right)+g_{\bar{\mu} \bar{\mu}, \mu \mu}^{*}\left(t_{3}\right)\right] .
\end{aligned}
$$




$$
\hat{G}_{\mu \nu, \mu^{\prime} \nu^{\prime}}\left(t_{2}\right) \equiv \mathrm{Q}_{\mu \nu} \hat{G}\left(t_{2}\right) \mathrm{Q}_{\mu^{\prime} \nu^{\prime}},
$$

which is again regarded as a conditional probability of finding the coherent state $\mu \nu$ at time $t_{2}$ when the initial coherence was $\mu^{\prime} \nu^{\prime}$ at $t_{2}=0$. We further introduce the traced EECT Green function as

$$
G_{\mu \nu, \mu^{\prime} \nu^{\prime}}\left(t_{2}\right) \equiv \operatorname{Tr}\left[(\mu \nu)^{\dagger} \hat{G}_{\mu \nu, \mu^{\prime} \nu^{\prime}}\left(t_{2}\right) \hat{\rho}_{\mu^{\prime} \nu^{\prime}}\right] .
$$

Here, $(\mu \nu)^{\dagger}$ is necessary to generate $\hat{\rho}_{\mu \nu}$ in the window function of Eq. (51). In our previous paper, we have derived the time-evolution equation for $G_{\mu \nu, \mu^{\prime} \nu^{\prime}}\left(t_{2}\right) .{ }^{19}$ In the present paper, we start again with Eq. (39) of Ref. 19,

$$
\begin{aligned}
& \frac{\partial \mathrm{Q}_{\mu \nu} e^{-i \hat{L} t} \mathrm{Q}_{\mu^{\prime} \nu^{\prime}} \rho(0)}{\partial t} \\
& =-\sum_{\chi \xi} \int_{0}^{t} d \tau \mathbf{Q}_{\mu \nu} \hat{L}_{1} \mathrm{P} \hat{L}_{1} \mathbf{Q}_{\chi \xi} e^{-i \hat{L} \tau} \mathbf{Q}_{\mu^{\prime} \nu^{\prime}} \rho(0),
\end{aligned}
$$

and rederive the time-evolution equation for $G_{\mu \nu, \mu^{\prime} \nu^{\prime}}\left(t_{2}\right){ }^{26}$ Tracing Eq. (56) over bath modes and assuming that the system and bath are initially uncorrelated $\hat{\rho}_{\mu^{\prime} \nu^{\prime}}=Q_{\mu^{\prime} \nu^{\prime}} \rho(0)$ $=\bar{\rho}_{\mu^{\prime} \nu^{\prime}}\left(\left\{q_{j}\right\}\right) \operatorname{Tr}_{\left\{q_{j}\right\}}\left\{\rho_{\mu^{\prime} \nu^{\prime}}(0)\right]$, we obtain

$$
\begin{aligned}
& \frac{\partial \operatorname{Tr}\left[(\mu \nu)^{\dagger} \mathrm{Q}_{\mu \nu} e^{-i \hat{L} t} \mathrm{Q}_{\mu^{\prime} \nu^{\prime}} \hat{\rho}_{\mu^{\prime} \nu^{\prime}}\right]}{\partial t} \\
& \quad=-\sum_{\chi \xi} \int_{0}^{t} d \tau \operatorname{Tr}\left[(\mu \nu)^{\dagger} \mathrm{Q}_{\mu \nu} \hat{L}_{1} \mathrm{P} \hat{L}_{1} \mathrm{Q}_{\chi \xi} e^{-i \hat{L} \tau} \mathrm{Q}_{\mu^{\prime} \nu^{\prime}} \hat{\rho}_{\mu^{\prime} \nu^{\prime}}\right] .
\end{aligned}
$$

Owing to this tracing over bath degrees of freedom, Eq. (57) is now independent of any bath modes and only includes system coherence information. Equation (57) with the definitions (54) and (55) leads to

$$
\frac{\partial G_{\mu \nu, \mu^{\prime} \nu^{\prime}}(t)}{\partial t}=\sum_{\chi \xi} \int_{0}^{t} d \tau K_{\mu \nu, \chi \xi} G_{\chi \xi, \mu^{\prime} \nu^{\prime}}(\tau),
$$

with the rate kernel function

$$
K_{\mu \nu, \chi \xi}=-\operatorname{Tr}\left[(\mu \nu)^{\dagger} \mathrm{Q}_{\mu \nu} \hat{L}_{1} \mathrm{P} \hat{L}_{1} \hat{\rho}_{\chi \xi}\right] .
$$

Here, on the right-hand side of Eq. (58), we divided the integrand into the rate kernel function and the EECT Green function. This derivation of Eq. (58) from Eq. (57) corresponds to the derivation of Eq. (A21) in Ref. 25 from Eq. (10) in Ref. 23 The physical validity of this approximation will be tested by checking whether the resultant kernel function for EECT is actually proportional to $\kappa T_{B}$ or not [see Eq. (63)]. We also mention that, once we have obtained Eq. (56), the final form of the time-evolution equation for EECT must have a form of Eq. (58), which is the non-Markov timeevolution equation with the time-independent kernel function. Due to the conservation of the total exciton number, we finally have the following time-evolution equation of $G_{\mu \nu, \mu^{\prime} \nu^{\prime}}(t)$ :

$$
\begin{aligned}
\frac{\partial G_{\mu \nu, \mu^{\prime} \nu^{\prime}}(t)}{\partial t}= & \sum_{\chi \xi} \int_{0}^{t} d \tau\left\{K_{\mu \nu, \chi \xi} G_{\chi \xi, \mu^{\prime} \nu^{\prime}}(\tau)\right. \\
& \left.-K_{\chi \xi, \mu \nu} G_{\mu \nu, \mu^{\prime} \nu^{\prime}}(\tau)\right\} .
\end{aligned}
$$

This equation is identical with Eq. (43) of Ref. 19. The explicit kernel function in Eq. (59) is calculated as

$$
\begin{aligned}
K_{\mu \nu, \chi \xi}= & -\delta_{\nu \xi}\left\langle q_{\mu \nu}^{(c)} q_{\nu \chi}^{(c)}\right\rangle+\delta_{\nu \chi}\left\langle q_{\xi \nu}^{(c)} q_{\mu \chi}^{(c)}\right\rangle \\
& +\delta_{\mu \xi}\left\langle q_{\xi \nu}^{(c)} q_{\mu \chi}^{(c)}\right\rangle-\delta_{\mu \chi}\left\langle q_{\xi \mu}^{(c)} q_{\mu \nu}^{(c)}\right\rangle,
\end{aligned}
$$

with

$$
\begin{aligned}
\left\langle q_{\xi \nu}^{(c)} q_{\mu \chi}^{(c)}\right\rangle & =\sum_{m} \phi_{\xi}(m) \phi_{\nu}^{*}(m) \phi_{\mu}(m) \phi_{\chi}^{*}(m)\left\langle q_{m m}^{(c)} q_{m m}^{(c)}\right\rangle, \\
& =\sum_{m} \phi_{\xi}(m) \phi_{\nu}^{*}(m) \phi_{\mu}(m) \phi_{\chi}^{*}(m) \ddot{g}_{m m, m m}(0) \sim \kappa T_{B} .
\end{aligned}
$$

Finally, we can calculate the three distinctively different contributions, i.e., $\quad R^{(0)}\left(t_{3}, t_{2}, t_{1}\right), \quad R_{\mathrm{EPT}}\left(t_{3}, t_{2}, t_{1}\right), \quad$ and $R_{\mathrm{EECT}}\left(t_{3}, t_{2}, t_{1}\right)$, which constitute the total nonlinear response function $R^{(3)}\left(t_{3}, t_{2}, t_{1}\right)$ in Eq. (18).

\section{IMPORTANT MATERIAL PARAMETERS FOR NUMERICAL CALCULATIONS OF B-DNA $(\mathrm{dA})_{10}-(\mathrm{dT})_{10}$}

Among various model DNA double helices, we shall focus on the $B$-form DNA consisting of ten deoxyadenosinethymidine base pairs, i.e., $(\mathrm{dA})_{10^{-}}(\mathrm{dT})_{10}$. Ten base pairs can be a minimal size DNA duplex system since one reel of a DNA duplex helix consists of about ten nucleoside pairs.

In $(\mathrm{dA})_{10^{-}}(\mathrm{dT})_{10}$, the intrastrand coupling constants between two dA and between two dT are $J_{m n}^{\text {intra }}=170$ and $217 \mathrm{~cm}^{-1}$, respectively. The interstrand coupling constant between pairing $\mathrm{dA}$ and dT bases is comparatively large and it was estimated as $J_{m n}^{\text {inter }}=248 \mathrm{~cm}^{-1}$ (see Tables V and VI of Ref. 3). The electronic coupling constant between dA in the $n$th base pair and dT in the $n \pm 1$ th base pair is $J_{m n}^{\text {inter }}$ $=80 \mathrm{~cm}^{-1}$.

Since a DNA duplex helix in solution is relatively flexible with some plasticity, the coupling constants may be distributed around the above average values, as shown in Refs. 6 and 11. Thus, for the numerical calculation, we will introduce the following off-diagonal disorders:

$$
f\left(J_{m n}\right)=\exp \left[-\frac{\left(J_{m n}-\widetilde{J}_{m n}\right)^{2}}{\vec{J}_{m n}^{2}}\right],
$$

where the standard deviation is a quarter of the average value, $\bar{J}_{m n}=0.25 \sqrt{2} \widetilde{J}_{m n}{ }^{6}{ }^{6}$ Note that the off-diagonal disorder $\bar{J}_{m n}$ was deduced from the numerical simulations of the DNA helices in solution. ${ }^{6}$

In addition, the diagonal disorder of base excitation energy should be taken into consideration, and its distribution is again specified by the Gaussian distribution 


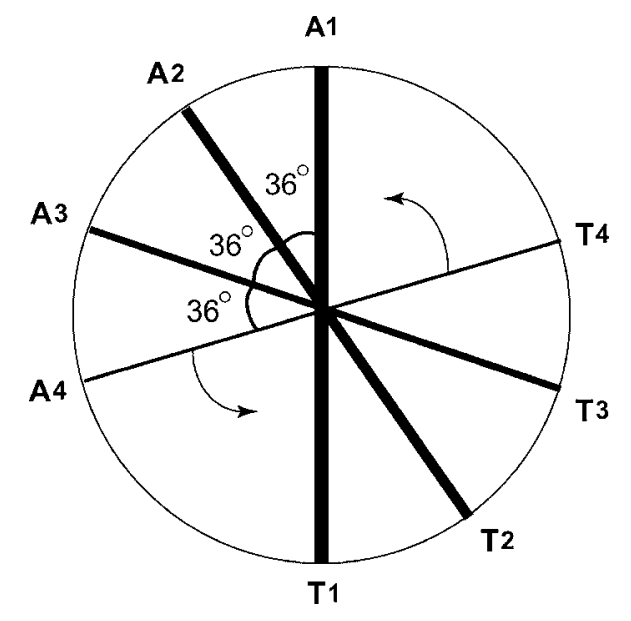

FIG. 4. Top view of the typical $B$-DNA structure. The subscripts express the order of ten nucleoside pairs. Each nucleoside pair twists just at $36^{\circ}$. The specific intra- and interstrand coupling constants are shown in Fig. 1.

$$
f\left(\Omega_{m}\right)=\exp \left[-\frac{\left(\Omega_{m}-\hat{\Omega}_{m}\right)^{2}}{\bar{\Omega}_{m}^{2}}\right],
$$

with the characteristic excitation frequency of $\mathrm{dA}, \hat{\Omega}_{m}$ $=38800 \mathrm{~cm}^{-1}$, and that of dT, $\hat{\Omega}_{m}=37500 \mathrm{~cm}^{-1}$. From the experimentally measured absorption spectra, the full widths at half maximum (FWHMs), $\sigma=2 \sqrt{\log 2} \bar{\Omega}_{m}$, of dA and dT are estimated to be 4000 and $5200 \mathrm{~cm}^{-1}$, respectively. ${ }^{3,12}$ The corresponding transition dipole moments have the absolute values $d_{A}=3.7$ and $d_{T}=3.68 \mathrm{D}$, respectively. Their transition dipole directions were experimentally determined (see Ref. 3 ) and are adopted in the present numerical calculation. As shown in Fig. 5 of Ref. 3, the electronic couplings, $J_{m n}^{\text {intra }}$ and $J_{m n}^{\text {inter }}$, strongly depend on the twist angle between two neighboring base pairs; the electronic couplings reach maximum values when the twist angle is $0^{\circ}$. As shown in Fig. 4, the coupling constants adopted in this paper correspond to those at the twist angle of $36^{\circ}$, which is the average twist angle for a typical $B$-form DNA. The above excitation energy of dA corresponds to the $S_{0} \rightarrow S_{2}$ transition energy, and the lowest transition, $S_{0} \rightarrow S_{1}$, is not included. This is because the transition dipole moment associated with the $S_{0} \rightarrow S_{2}$ transition is much larger than that of the $S_{0} \rightarrow S_{1}$ transition and, as shown in Fig. 2 of Ref. 3, $S_{0} \rightarrow S_{2}$ transition of dA essentially determines the linear absorption spectrum of $\mathrm{dA}^{3,6}$

As shown in Eq. (44), the spectral density representing the base-bath interaction is specified by two parameters, that is, the relaxation time of noise correlation $\tau_{B}$ and the solvent reorganization energy $\kappa$. We assume that $\tau_{B}=50 \mathrm{fs}$ and $\kappa$ $=5320 \mathrm{~cm}^{-1}$, and the temperature for the present numerical calculations is set to be $T_{B}=295 \mathrm{~K}$. As will be shown in the next section, we have confirmed that the experimentally measured linear absorption and steady-state fluorescence spectra are quantitatively well reproduced with the important material parameters mentioned in this section.

\section{TIME-DEPENDENT FLUORESCENCE SPECTROSCOPY (TDFS)}

One of the most direct ways to probe the excited-state relaxation processes is to measure time-dependent changes of fluorescence spectra by employing a femtosecond laser pulse excitation and subsequent time resolution of fluorescence spectra. For example, the time-dependent fluorescence Stokes shift measurement has been proved to be extremely useful to study ultrafast solvation dynamics of a chromophore in a condensed phase. Unlike simple dye molecules, which are modeled as a two-level system, DNA duplex helix is a coupled multichromophore system, so that not only the chromophore-bath interaction-induced fluorescence Stokes shift but also the exciton relaxation should contribute to the TDFS.

The TDFS $\sigma_{f}\left(\omega, t_{2}\right)$, where $\omega$ is an emission frequency and $t_{2}$ is the delay time between the excitation pulse and fluorescence, can be written as

$$
\begin{aligned}
\sigma_{f}\left(\omega, t_{2}\right)= & \mathrm{R} e \int_{0}^{\infty} d t e^{i \omega t_{3}} i\left\{R_{I}\left(t_{3}, t_{2}, 0\right)+R_{\mathrm{EPT}}\left(t_{3}, t_{2}, 0\right)\right. \\
& -R_{\mathrm{EPT}}\left(t_{3}, 0,0\right)+R_{\mathrm{EECT}}\left(t_{3}, t_{2}, 0\right) \\
& \left.-R_{\mathrm{EECT}}\left(t_{3}, 0,0\right)\right\} .
\end{aligned}
$$

The first term given in Eq. (20) describes the time-dependent solvation dynamics when there is neither EPT nor EECT processes. The expression for this term is not given in the doorway-window picture, and the correlation between the initially created and time-evolved coherence state during $t_{1}$ and the second electronic coherence state evolution during $t_{3}$, i.e., spectral diffusion, is correctly taken into consideration. When EPT and EECT processes are negligibly slow in comparison with the solvation dynamics, the first term becomes dominant in determining TDFS like a simple two-level chromophore system. However, as will be shown below, the time scales of EPT and EECT processes are comparable to that of the solvation dynamics, so that the other terms in Eq. (66) are to be included to quantitatively describe the TDFS. The second term given in Eq. (31) describes the contributions from EPT to the TDFS and its explicit expression is given as

$$
\begin{aligned}
R_{\mathrm{EPT}}\left(t_{3}, t_{2}, 0\right)= & -i \sum_{\mu, \nu}\left|d_{\mu}\right|^{2}\left|d_{\nu}\right|^{2} G_{\mu \mu, \nu \nu}\left(t_{2}\right) \\
& \times \exp \left[i \epsilon_{\mu} t_{3}-g_{\mu \mu, \mu \mu}\left(t_{3}\right)-2 i \kappa_{\mu \mu, \mu \mu} t_{3}\right],
\end{aligned}
$$

from Eq. (30) with the doorway and window functions [Eqs. (34) and (35)]. The EPT from $\mu \mu$ to $\nu \nu$ during $t_{2}$ is fully described by the EPT Green function, $G_{\mu \mu, \nu \nu}\left(t_{2}\right)$, whose time evolution is determined by the quantum master equation [Eq. (38)]. Similarly, the fourth term in Eq. (66) describes the EECT contributions which is defined in Eq. (49). It should be noted that the third and the fifth terms in Eq. (66) are required because there is neither EPT nor EECT at $t_{2}=0$, so that the initial TDFS, $\sigma_{f}(\omega, 0)$, should be identical to the first term in Eq. (66) only. Those terms are identical to the last terms in Eqs. (31) and (49), respectively. Additionally, we found that the last two terms of Eq. (66) vanish in the case of $t_{1}=0$ for any $t_{2}$. This indicates that the TDFS is not affected by the EECT processes, so that the TDFS measurement is determined only by solvation dynamics and the EPT contribution. We finally note that TDFS does not include contributions from the ground-state bleaching and excited-state ab- 
sorption. In our numerical calculation of the TDFS, we excluded $R_{\mathrm{II}}\left(t_{3}, t_{2}, t_{1}\right)$ and $R_{\mathrm{III}}\left(t_{3}, t_{2}, t_{1}\right)$ in Eq. (19) and also the corresponding two-exciton contributions in the window functions of EPT and EECT shown in Eqs. (35) and (53), respectively. Note that $R_{\mathrm{II}}\left(t_{3}, t_{2}, t_{1}\right)$ and $R_{\mathrm{III}}\left(t_{3}, t_{2}, t_{1}\right)$ describe the Liouville paths including the ground-state bleaching and excited-state absorption effects, respectively. ${ }^{25}$

Using Eq. (66) and the material parameters for $(\mathrm{dA})_{10^{-}}(\mathrm{dT})_{10}$ given in Sec. V, we carried out the numerical calculations of the TDFS and the graphical results are plotted in Fig. 5. The present results were obtained by taking an average over 1000 realizations of the diagonal and offdiagonal disorders. Figure 5(a) includes both solvation dynamics and EPT, but Fig. 5(b) does not include the latter. The time-dependent changes of the TDFS are extremely fast with or without the EPT. The lowest figure in Fig. 5 depicts the time-dependent fluorescence Stokes shift. Although the Stokes shifts are almost completed within $200 \mathrm{fs}$, these ultrafast fluorescence Stokes shifts largely depend on the relaxation time $\tau_{B}$ that was assumed to be $50 \mathrm{fs}$ in the present paper. While $\tau_{B}$ is now an undetermined parameter, our results indicate a possibility to determine the characteristic time scale $\tau_{B}$ in the $B$-form DNA system by means of the TDFS measurement. In this regard, it will be highly interesting to carry out a TDFS experiment on a DNA duplex helix system in the future. Nevertheless, it is clear that, once the EPT contribution is included, the magnitude of the fluorescence Stokes shift becomes larger than that without the EPT process.

In Figs. 5(a) and 5(b), the spectrum at $t_{2}=0$ fs (the dotted line) should be identical to the absorption spectrum and its lineshape is actually in good agreement with the experimental results. ${ }^{6,14}$ For example, the maximum frequency and FWHM of the dotted line are 258 and $35.5 \mathrm{~nm}$, while those of the experimental results are 258 and $39 \mathrm{~nm}$, respectively. The steady-state fluorescence spectrum corresponds to the converged TDFS at 150 fs (the solid line) in Fig. 5(a) and it quantitatively agrees with the experimentally measured spectrum in Ref. 14. Due to the distributions of the excitonic states and their dipole strengths, the steady-state fluorescence spectrum is asymmetric. If the present system were a simple two-level system, the steady-state fluorescence spectrum would be a mirror image of the absorption spectrum. However, since the $B$-DNA we study is a coupled multichromophore system consisting of the 20 excitonic states with varying dipole strengths and transition frequencies, the present results are deviated from the simple mirror profile. From the above comparisons, the present material parameters adopted to construct the $B$-DNA excitation Hamiltonian seem reasonable and useful to reproduce the experimentally reported linear absorption and steady-state fluorescence spectra of the $B$-DNA duplex helix system.

\section{PHOTON ECHO SPECTROSCOPY}

Although the time-resolved fluorescence spectroscopy is a valuable means to study dynamical properties of excited states, it requires an upconversion technique with a timegating pulse if the time scale of the dynamics is subpicosec- (a)

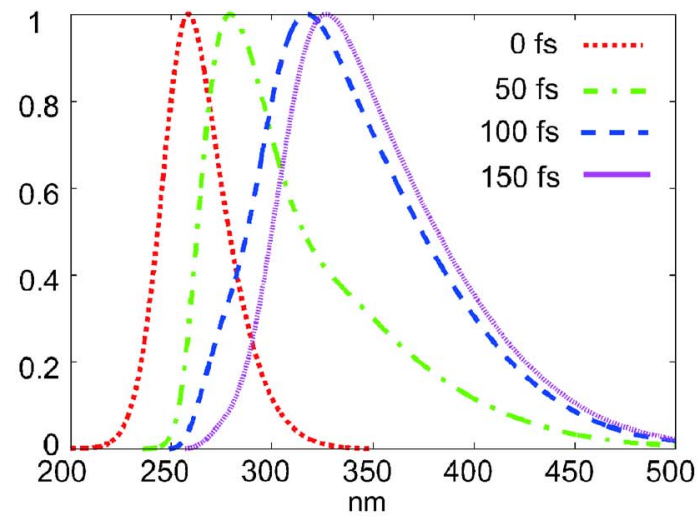

(b)
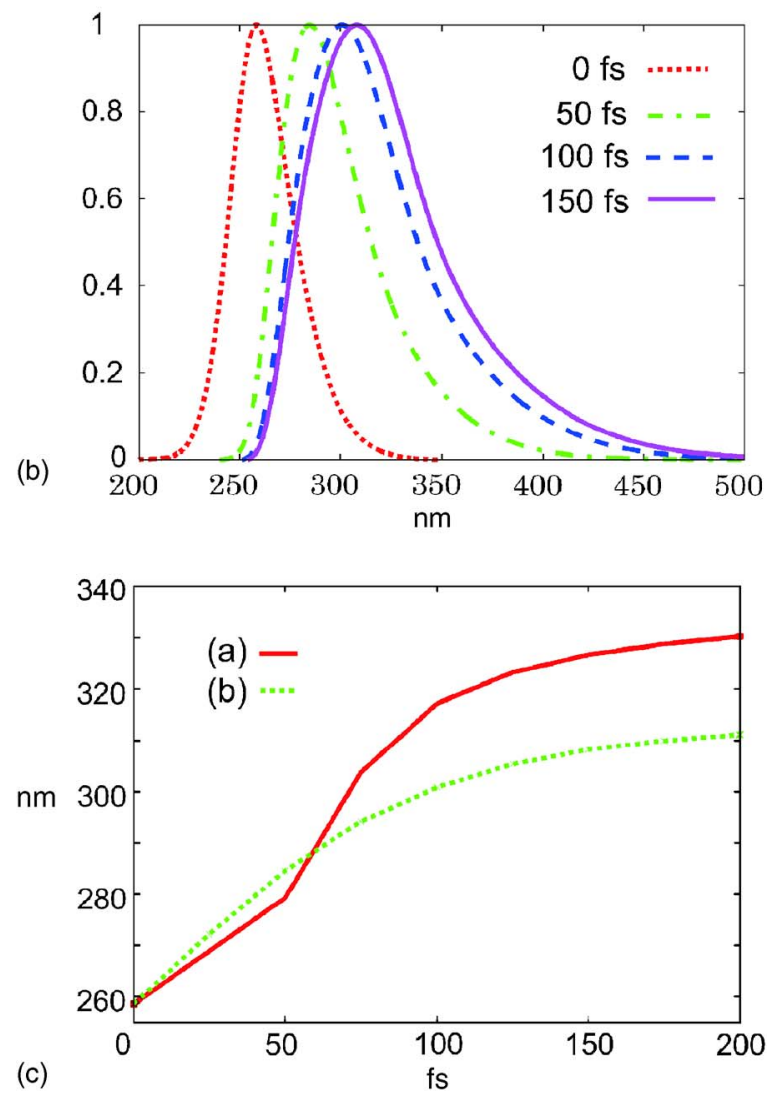

FIG. 5. (Color online) Time-dependent fluorescence spectra of $(\mathrm{dA})_{10^{-}}(\mathrm{dT})_{10}$ with (a) and without (b) the EPT contribution. The dotted lines correspond to the absorption spectrum and is in accord with the experimental result, Fig. 12 of Ref. 6. The converged spectra shown as the solid lines should be the steady-state fluorescence spectrum of $(\mathrm{dA})_{10^{-}}(\mathrm{dT})_{10}$ which is actually in harmony with the experimental result, Fig. 4 of Ref. 4. The direct experimental observable, the peak frequency vs time, is shown in the lowest figure (c). The different decaying curves, the solid and dotted lines, directly illustrate the different time scales of the solvent relaxation only and the additional EPT contribution, respectively.

ond. Recently, the photon echo spectroscopy utilizing multiple femtosecond pulses has been widely used to study such femtosecond dynamics of not only electronically excited states but also ground-state bleaching and excited-state absorption processes of coupled multichromophore systems. In this section, the numerically calculated photon echo signals of the $B$-form DNA model system will be presented and discussed in detail. The photon echo signals calculated in this paper were obtained by averaging over 1000 realizations of the diagonal and off-diagonal disorders, which is sufficient to 
converge the present ultrafast signals. The rotational average of the fourth-rank tensorial nonlinear response function of randomly oriented DNA molecules in solution was also performed and only the $[Z Z Z Z]$ tensor component will be discussed here; the $[Z Z Z Z]$ component corresponds to the case when three incident electric fields and its echo signal field have polarization directions along the $Z$ axis in a space-fixed frame and their propagation directions are perpendicular to the $Z$ axis. Hereafter, the photon echo signals are normalized to make the maximum value unity. We will discuss the following three different cases to examine the effects of EPT and EECT on the photon echo signals separately:

(A) total photon echo signal with both EPT and EECT contributions,

(B) photon echo signal without EECT, and

(C) photon echo signal without both EPT and EECT.

Therefore, any differences among the photon echo signals for these three cases should be attributed to one or both of the EPT and EECT processes; we can discuss roles of EPT and EECT in the excited energy dynamics of DNA duplex through the changes appearing in the calculated photon echo spectra.

\section{A. 2D Time-resolved photon echo (TRPE) and PEPS}

As shown in Sec. III, in the impulsive limit, the timeresolved heterodyne-detected photon echo signal is linearly proportional to the associated nonlinear response function. For a fixed second delay time $t_{2}$, the signal is a $2 \mathrm{D}$ function with respect to $t_{1}$ and $t_{3}$. In particular, the absolute magnitudes of the photon echo signals $I_{1}\left(t_{1}, t_{2}, t_{3}\right)=\left|R^{(3)}\left(t_{3}, t_{2}, t_{1}\right)\right|$ are plotted in Fig. 6 for $t_{2}=0-57.5$ fs from top to bottom.

The figures in the left column are the absolute magnitude spectra for case (A). The initial echo signal is diagonally elongated, indicating the coherence between the excitation and emission states in time domain. This demonstrates the slow modulations of bath modes in the $B$-form DNA duplex system. However, as $t_{2}$ increases, the elongation is suppressed due to the memory loss and the diagonally elongated main peak becomes uniform like a peak in the case of fast modulation of bath modes. Its time scale is shorter than that of the light-harvesting complex II (LH2) antenna system partly because the relaxation time $\tau_{B}$ is now assumed to be shorter than that of LH2; $\tau_{B}$ should be experimentally determined in the near future. ${ }^{19}$ On the other hand, the second peak appears as $t_{2}$ increases. We found that this peak is attributable to the coexistence (interference) of the contributions from the ground population state and excited-state absorption to the nonlinear response function. The former effect is included in $R_{\mathrm{II}}\left(t_{3}, t_{2}, t_{1}\right)$, while the latter effect appears in $R_{\mathrm{III}}\left(t_{3}, t_{2}, t_{1}\right), R_{\mathrm{EPT}}\left(t_{3}, t_{2}, t_{1}\right)$, and $R_{\mathrm{EECT}}\left(t_{3}, t_{2}, t_{1}\right)$. Indeed, once we neglect one of these two contributions, the second peak disappears and there is only one main peak. The coherence between the contributions including the ground population state and excited-state absorption decays slower than the others, which leads to the apparent second peak against the main peak as $t_{2}$ increases.

Comparing the 2D TRPE signals for the three cases (A)-
(C), we found that the EPT and EECT affect the photon echo signals. First, the main peaks without the EECT process have the stronger coherence compared to that of case (A). The main peaks in cases (B) and (C) are still elongated and exhibit some coherence behavior even at $t_{2}=57.5 \mathrm{fs}$. It can be concluded that, owing to EECT, the ultrafast decoherence has been achieved in $(\mathrm{dA})_{10^{-}}(\mathrm{dT})_{10}$. In addition, the second peak becomes smaller due to the EPT contribution; the second peak in case (B) is smaller than that in case $(C)$. The EPT mixes the two Liouville paths of the ground population state and excited-state absorption and reduces the coexistence effect. Note that the EECT process makes the main peak decay much faster, which results in the comparatively larger second peak in case (A) than that in case (B). Additionally, being compared to the main and second peaks in case (A), those in cases (B) and (C) are more shifted from the diagonal axis; the main and second peaks interfere with each other, which leads to the simultaneous shift. As well as in case (A), once we neglect one of the contributions from the ground-state bleaching and excited-state absorption, the second peaks in cases (B) and (C) disappear and we have only one diagonal peak in the each case. Therefore, the more shifted peaks in cases (B) and (C) can be explained by concluding that EECT can reduce the coexistence effect.

Although the 2D TRPE spectra in Fig. 6 contain indispensable information, it has been found that PEPS, which is a peak position of an integrated photon echo signal as a function of $t_{1}$, can provide more direct information on a time scale of excited-state solvation dynamics and other underlying dynamic processes. ${ }^{20}$ The time-integrated photon echo signal is obtained by

$$
I_{2}\left(t_{1}, t_{2}\right)=\int_{0}^{\infty}\left|R^{(3)}\left(t_{3}, t_{2}, t_{1}\right)\right|^{2} d t_{3} .
$$

If there is short-time inhomogeneity of the base transition energies, the echo signal $I_{2}\left(t_{1}, t_{2}\right)$ initially increases with $t_{1}$, reaches a maximum value, and then decays in time $t_{1}$. The peak position denoted as $t_{1}^{*}\left(t_{2}\right)$ is a function of $t_{2}$. The calculated PEPS is drawn in Fig. 7.

At time $t_{2}=0$, the PEPS values are just about $3.5 \mathrm{fs}$ for all the $(\mathrm{A})-(\mathrm{C})$ cases. However, their decaying patterns are heavily dependent on whether the EECT process is taken into account or not. The PEPS with EECT decays to zero before $40 \mathrm{fs}$, while the coherence persistently survives when the EECT is not included. This demonstrates that the decoherence or memory loss rate is on the order of $50 \mathrm{fs}$ and is strongly influenced by the EECT contribution. We suggest that the EECT during $t_{2}$ can induce the ultrafast memory loss of the multiple quantum coherence created by the fieldmatter interactions. The zero PEPS in case (A) is related to the relatively large homogeneous parameter $\kappa$, which determines the absolute magnitude of base-phonon coupling strength and also the rate kernel function of EECT; ${ }^{19}$ the static inhomogeneity introduced by the diagonal and offdiagonal disorders is almost balanced by the EECT process. In fact, as shown in Fig. 6(a), the diagonally elongated main 
(A)
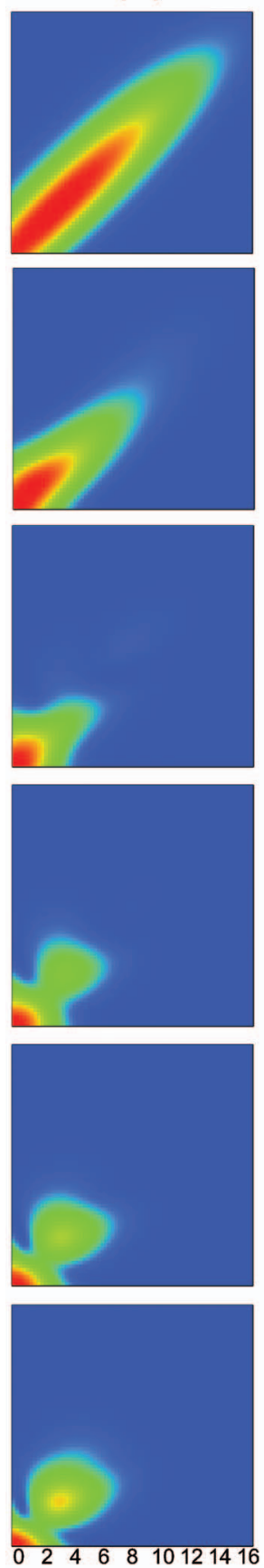

(B)
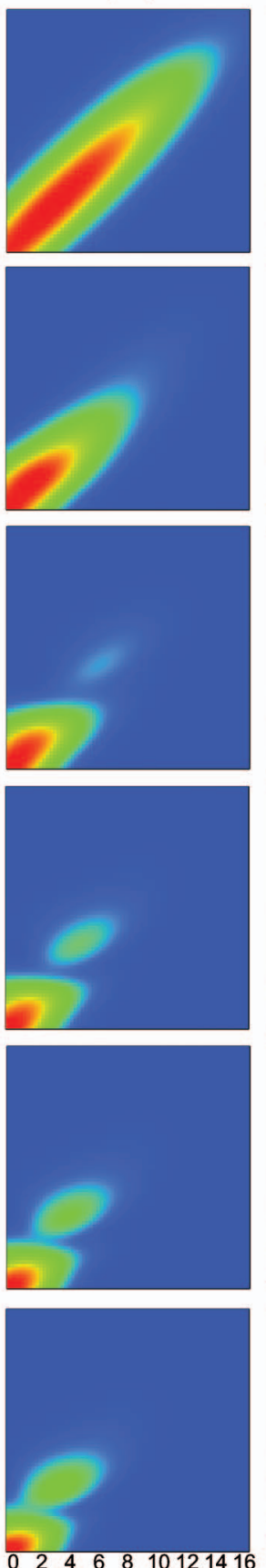

$t_{1}$ (fs)
(C)
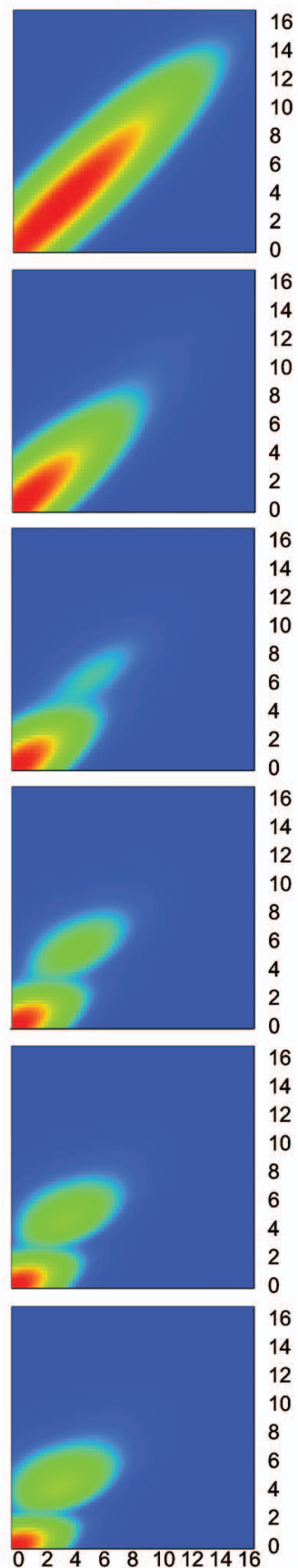

$02246810121416^{0}$ $t_{3}(\mathrm{fs})$

FIG. 6. (Color) TRPE signals $\left|R^{(3)}\left(t_{3}, t_{2}, t_{1}\right)\right|$ vs $t_{1}$ and $t_{3}$ with $t_{2}=0,11.5,23,34.5,46$, and 57.5 fs (from the upper figure to the lower figure, respectively). The left, middle, and right figures show cases (A), (B), and (C), respectively. peak becomes like a triangle-shape peak in the fast modulation case. We mention that, although the PEPS for case (A) converges to zero, not all the coherence was destroyed by the EECT process; the second peak appears in the lower figures of Fig. 6(a) as $t_{2}$ increases and $I_{2}\left(t_{1}, t_{2}\right)$ as a function of $t_{1}$ becomes to double peaked in case $(\mathrm{A})$ as $t_{2}$ increases. Similarly, the EPT process whose rate kernel function also depends on $\kappa$ reduces the effect of the inhomogeneity, as can be seen from the PEPSs of case (B) (the dotted line) and case (C) (the dashed line) in Fig. 7. However, its reduction is rather small and the PEPS for case (B) does not converge to zero.

\section{B. 2D photon echo spectra}

We finally calculate the 2D photon echo spectra by taking the double Fourier transformation of the 2D TRPE signals,

$$
\begin{aligned}
I\left(\Omega_{1}, t_{2}, \Omega_{3}\right)= & \int_{0}^{\infty} \int_{0}^{\infty} \exp \left(i \Omega_{1} t_{1}\right) \\
& \times \exp \left(-i \Omega_{3} t_{3}\right) R^{(3)}\left(t_{3}, t_{2}, t_{1}\right) d t_{1} d t_{3} .
\end{aligned}
$$

Figure 8 depicts the absolute magnitude spectra as a function of $\Omega_{1}$ and $\Omega_{3}$ that are conjugate Fourier frequencies of $t_{1}$ and $t_{3}$, respectively. 


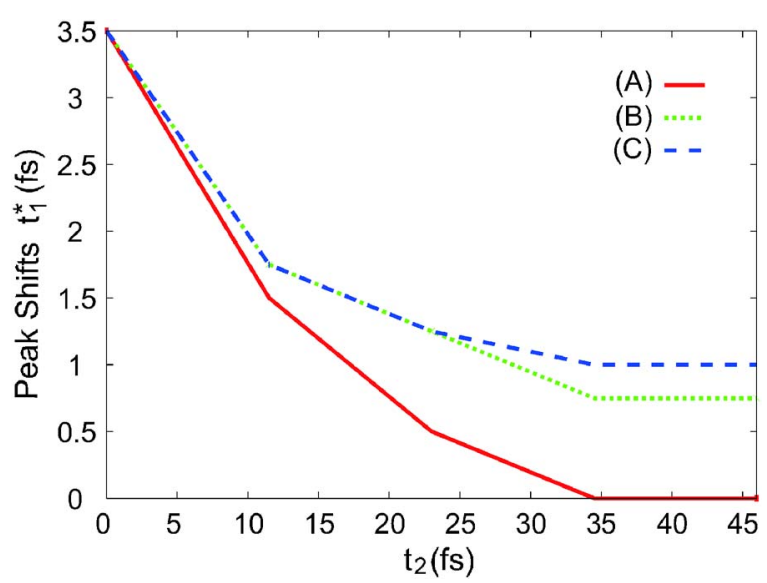

FIG. 7. (Color online) PEPSs $t_{1}^{*}\left(t_{2}\right)$ estimated from the time-integrated photon echo signals [Eq. (68)] with respect to $t_{2}$. The solid, dotted, and dashed lines indicate PEPS of cases (A), (B), and (C), respectively. Their direct comparison independently shows the effects of EPT and EECT.

At $t_{2}=0$, the $2 \mathrm{D}$ photon echo spectra are diagonally elongated regardless of cases $(\mathrm{A})-(\mathrm{C})$, indicating the preservation of memory of the transition frequencies between the coherence evolution periods $t_{1}$ and $t_{3}$. As $t_{2}$ increases (from top to bottom in Fig. 8), the 2D photon echo spectra dramatically change. Particularly, when we include the EECT contribution [Fig. 8(a)], the 2D spectrum at $t_{2}=57.5 \mathrm{fs}$ becomes a round shape along the diagonal. This suggests that the EECT processes are quite efficient to mix the transition frequencies and to induce the memory loss. Actually, the main broad peak in Fig. 8(a) appears in the frequency range between the two separated peaks in Figs. 8(b) and 8(c), which shows that the EECT contribution can mix the exciton transition energies. On the other hand, as can be seen in Figs. 8(b) and 8(c), the EPT process does not make any notable difference in the time evolution of the $2 \mathrm{D}$ photon echo spectrum. The small peak below the main peak corresponds to the contribution producing the TDFS in Fig. 5. The frequency of the small peak is lower than the corresponding peak of the TDFS, which is attributable to the additional contribution from EECT; although EECT does not contribute to the TDFS, the 2D photon echo spectra are influenced by EECT due to nonzero $t_{1}$ (see also Sec. VI).

Furthermore, we found that the EECT causes the complicated frequency dispersion of the peaks in subpicosecond time scales. The dispersed peaks at 23 and 34.5 fs originate from the presence of different electronic coherence states on the one-exciton state manifold. However, such dispersed and scattered peaks become merged into two broad peak at about $57.5 \mathrm{fs}$, which indicates that the EECT processes are completed in such a short time scale. This ultrafast dispersion and unifying may be attributed to the appearance of the second peak in Fig. 6(a). In fact, if one of the contributions from the ground-state bleaching and the excited state absorption is excluded, the frequency dispersion does not occur and only one main peak appears (data not shown here). The observation made here might provide an important clue on the excited state energy dynamics in DNA duplex helices and have some biological implications. In order to avoid photodamage on natural DNA duplex, biological systems might have evolved in such a way to get the stable and effective energy dissipation and intramolecular energy transfer mechanisms. In another words, the energy diffusion in a DNA helix could occur through a few specific excitation energy transfer channels and pathways in such an ultrafast time.

\section{CONCLUDING REMARKS}

The ultrafast exciton dynamics in the DNA duplex helices have been theoretically described and the contributions from the EPT and EECT processes to the nonlinear response function associated with ultrafast nonlinear optical spectroscopy have been studied in detail. Developing the appropriate DNA exciton Hamiltonian model, we could calculate the exciton population and coherence transfer Green functions by solving their non-Markov time-evolution equations. In order to test the validity of the material parameters determined and used in the present work, we directly compared the experimentally measured absorption and steady-state fluorescence spectra of the $B$-form double helix in water solvent with our numerically calculated spectra of the $(\mathrm{dA})_{10^{-}}(\mathrm{dT})_{10}$ system. Although the experimentally measured absorption and fluorescence spectra of $(\mathrm{dA})_{10^{-}}(\mathrm{dT})_{10}$ are not available, they are not strongly dependent on the number of base pairs except for some impurity artifacts of the sample. ${ }^{14}$ Here, the resultant linear spectra calculated were found to be in good agreement with the corresponding experimental spectra. However, it should be mentioned that the bath relaxation time constant $\tau_{B}$ could not be determined in a self-consistent way because there is no femtosecond TDFS or nonlinear optical spectroscopy that can provide information on such nucleoside-bath interaction dynamics. In contrast, the relaxation time of the LH2 system was estimated through its PEPS experiment. ${ }^{30}$ Consequently, once the relaxation time for the DNA duplex system is measured, it will become possible to calculate the exciton dynamics in various DNA helices by our present theory. Similarly, once PEPS of a DNA duplex is measured, we can estimate the time after which coherences almost vanish and EECT essentially stops contributing to the nonlinear spectra, as discussed in the case of the LH2 system. ${ }^{1,26,29}$ Nevertheless, we have theoretically shown that the ultrafast dynamics of DNA excitons are quite significant and can be studied by the nonlinear optical spectroscopy such as the PEPS and photon echo spectroscopy. We have fully calculated the nonlinear response functions including the EPT and EECT contributions which can be directly measured in the nonlinear optical experiments. In addition, the TDFS and photon echo spectra of the $B$-DNA duplex helix, $(\mathrm{dA})_{10^{-}}(\mathrm{dT})_{10}$, were numerically calculated. The TDFS demonstrated that the EPT process induces the spectral diffusion on the excitonic state manifold and that the time-dependent Stokes shift is completed in subpicoseconds. The PEPS of the $B$-DNA duplex helix also decays to zero in less than $50 \mathrm{fs}$, indicating an ultrafast memory loss induced by the EECT process on the manifold of delocalized excitonic states. The 2D TRPE and photon echo spectra were calculated with and without the EPT and EECT processes to investigate the significant spectroscopic signatures of such exciton dynamics in the DNA duplex system. We found that the 
(A)
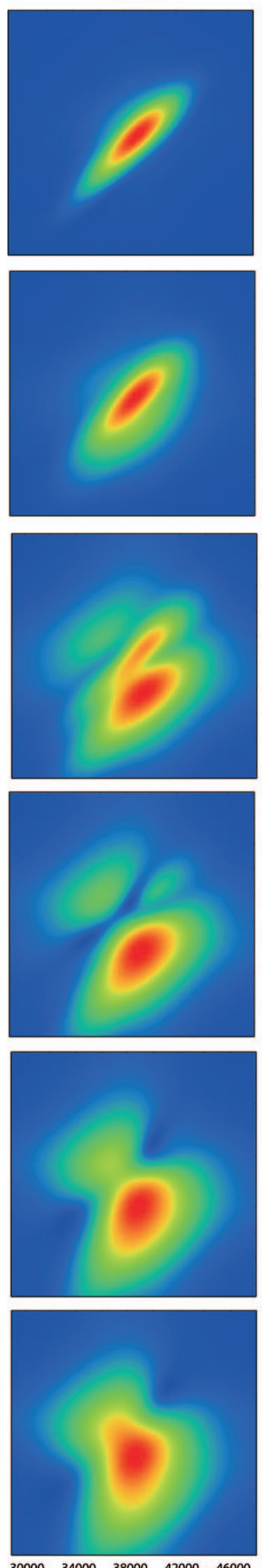

$30000 \quad 34000 \quad 38000 \quad 42000 \quad 46000$
(B)
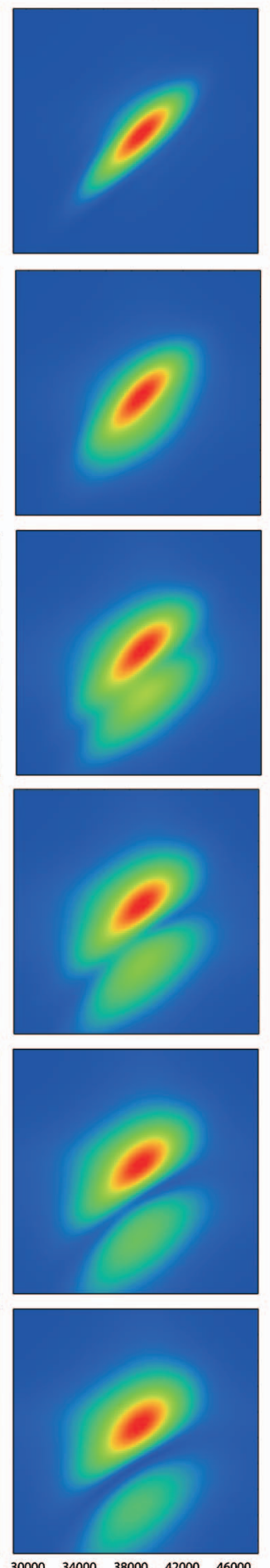

$\Omega_{1}\left(\mathrm{~cm}^{-1}\right)$
(C)
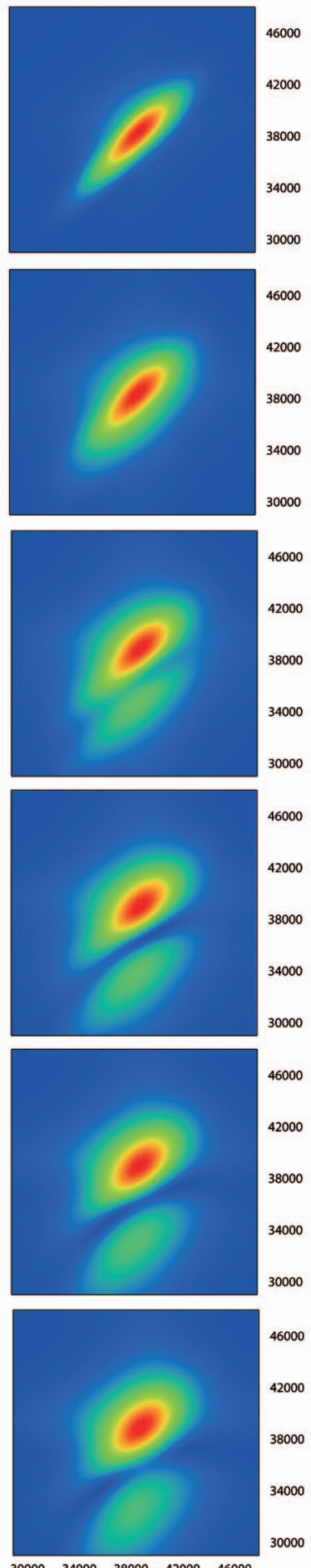

$30000 \quad 34000 \quad 38000 \quad 42000 \quad 46000$

\section{$\Omega 3\left(\mathrm{~cm}^{-1}\right)$} $2 \mathrm{D}$ photon echo spectra $I\left(\Omega_{1}, t_{2}, \Omega_{3}\right)$ at $t_{2}=0,11.5,23,34.5,46$, and 57.5 fs (from the upper figure to the lower figure, respectively). The horizontal and vertical axes are $\Omega_{1}$ and $\Omega_{3} \mathrm{~cm}^{-1}$, respectively. The left, middle, and right figures show the cases (A), (B), and (C), respectively. diagonally elongated 2D TRPE spectrum at $t_{2}=0$ fs becomes broader and uniform as $t_{2}$ increases and that the 2D photon echo spectrum at $t_{2}=0 \mathrm{fs}$ becomes almost round along the diagonal axis within subpicoseconds. Such dramatic spectral shape changes are largely due to the EECT process. We thus conclude that the exciton transfers, especially the EECT process, induce the ultrafast decoherence, memory loss, and frequency diffusion of the multiple quantum coherences created by the field-matter interactions. We call this rapid frequency diffusion as hopping dephasing in order to distinguish it from usual pure dephasing. The hopping dephasing induced by EECT differs from the conventional pure dephasing. The latter makes the amplitude of a given coherence monotonically decrease in time, whereas EECT can have a backward transfer process since EECT involves hopping among strongly correlated close coherences. Because of the rapid hopping 
motions among such close coherences induced by the exciton-phonon interactions, only the hopping dephasing can achieve such ultrafast quantum decoherence.

In the present work, we have adopted the exciton representation to describe the electronically excited states of the DNA duplex helix. However, the excitonic state is not necessarily a fully delocalized state. One of the most important conditions for exciton formation is the strong interbase electronic couplings in comparison with the base-phonon couplings, which leads to the independent excited-state transfer rate from the interbase couplings. ${ }^{23}$ Once this condition is satisfied, the DNA electronic Hamiltonian matrix can be diagonalized to recast it as the DNA exciton Hamiltonian. The degrees of delocalization for different excitonic states are not uniform and determined by the relative strengths of the interbase couplings as well as by the diagonal and off-diagonal disorders. Actually, although the above condition is satisfied in the DNA duplex helix, ${ }^{16}$ the excitonic states are delocalized over not the entire constituent bases but several bases in the DNA duplex. Nevertheless, this incomplete delocalization ensures the perturbative treatment of the off-diagonal exciton-phonon couplings. ${ }^{23}$ This is because $q_{\mu \nu}^{(c)}$ $=\Sigma_{m} \phi_{\mu}(m) \phi_{\nu}^{*}(m) q_{m m}^{(c)}$ are generally small due to such incomplete delocalization even when the base-phonon coupling constants $q_{m m}^{(c)}$ are large, while the diagonal exciton-phonon couplings are independent of the degrees of delocalization. Since biomolecules usually have disorders, the excitonic states are not completely delocalized and thus the present exciton transfer theory that treats $q_{\mu \nu}^{(c)}$ as perturbation terms can be proper to study ultrafast excited-state dynamics in such biomolecules.

EECT is not a simple relaxation process of coherence but an ultrafast exciton hopping transfer described by the non-Markov time-evolution equation [Eq. (60)] where not only forward but also backward transfer is taken into account. Such ultrafast exciton hopping transfers can be seen in B850 LH2 antenna, as shown recently in Ref. 19, and they can also play an important role in DNA duplex systems. In order to derive Eqs. (38) and (60) and to make a clear connection of them to the Redfield theory, let us start from the following quantum Liouville equation:

$$
\frac{\partial \rho(t)}{\partial t}=-i \hat{L} \rho(t) .
$$

Using the relation $P+Q=1$, the above equation can be divided into the following two equations:

$$
\frac{\partial \mathrm{P} \rho(t)}{\partial t}=-i \mathrm{P} \hat{L} \mathrm{P} \rho(t)-i \mathrm{P} \hat{L} \mathrm{Q} \rho(t)
$$

and

$$
\frac{\partial \mathrm{Q} \rho(t)}{\partial t}=-i \hat{\mathrm{L}} \mathrm{Q} \rho(t)-i \mathrm{Q} \hat{L} \mathrm{P} \rho(t)
$$

Equations (38) and (60) are obtained from Eqs. (71) and (72), respectively. For example, the solution of Eq. (71),

$$
\mathrm{P} \rho(t)=-i \int_{0}^{t} d \tau e^{-i(t-\tau) \mathrm{P} \hat{L}} \mathrm{P} \hat{L} \mathrm{Q} \rho(\tau)+e^{-i t \operatorname{P} \hat{L}} \mathrm{P} \rho(0),
$$

is inserted into the second term on the right-hand side of Eq. (72), and the second term indeed plays an important role in essentially describing the EECT process [Eq. (60)], as explained in Ref. 19. In the case of the Redfield theory with the secular approximation, the time evolution of coherence is determined by the first term on the right-hand side of Eq. (72) [see Eq. (8) and the following explanation in Ref. 31]. On the other hand, the time-evolution equations for EPT and EECT [Eqs. (38) and (60)] include the contributions from the second terms on the right-hand sides of Eqs. (71) and (72), respectively. Actually, Novoderezhkin et al. who studied the B800-B850 light-harvesting antenna complex of a purple bacterium also wrote in Ref. 32 that the modified Redfield theory is more general than the usual Redfield theory. However, they did not use the modified Redfield theory since there was only the time-evolution equation for EPT [Eq. (38)] at that time. In addition, as indicated in Refs. 23 and 32 , one should be very careful about the applicability of the Redfield theory to ultrafast exciton transfers in biocomplexes such as the LH2 antenna system. As shown in Ref. 32, even the nonsecular Redfield theory cannot reproduce the experimental spectroscopic data in subpicoseconds.

We further discuss some differences between the Redfield theory and the present exciton transfer theory of EPT and EECT processes. First, the bath correlation function is time evolved with an exciton-dependent phonon Hamiltonian in the present theory since the diagonal exciton-photon coupling terms are nonperturbatively included in the present exciton theory. On the other hand, as indicated in Sec. 3.1 of Ref. 23, all excitons have the same potential energy surface in the Redfield theory. Second, owing to the shifted potential energy of the acceptor exciton from that of the donor exciton by reorganization energy associated with the nonperturbative diagonal exciton-phonon couplings, an exciton transfer between two different excitons with a large energy gap at zeroorder level can occur. In contrast, such excitation transfer cannot be described by the Redfield theory unless a highfrequency vibrational phonon mode is invoked to compensate the large energy gap between the donor and acceptor exciton states. Finally, the conventional Redfield theory is based on the assumption that the density matrix does not change significantly during times of the relaxation time of phonon bath correlation. ${ }^{33}$ This limitation in the Redfield theory might conflict with some ultrafast exciton dynamics found in biomolecules. ${ }^{32}$

If a charge is injected to a DNA helix as in Ref. 34, or if a photosensitizer and an electron donor are added to a DNA helix, or if intensive UV light is radiated to a DNA helix for a long time, charge transfers would be important and would be completed with the nonlinear optical processes considered here. However, our model system does not have any electron donors artificially added to nor any external charges are injected to the system. Consequently, such a charge transfer process might not be an effective and relevant process in the present model DNA system. In fact, as introduced in Sec. I, almost all the previous works on electronically excited nor- 
mal DNA duplex helices did not discuss about such charge transfer effects. Furthermore, since the excitation transfer rates are not proportional to the square of the electric couplings between bases, the transfer processes cannot be explained by the Förster theory nor the electron transfer theory; their transfer rates are generally both proportional to the square of the electronic couplings between bases. ${ }^{16}$ This indicates that the exciton representation can be an appropriate picture for the excited state of DNA double helix and that the excitation transfer processes should be described as exciton migrations on an excited-state manifold constructed by delocalized exciton states. This and our work show that the exciton representation of excited states of a DNA duplex can be an appropriate picture and that the excitation energy transfer processes should be described as exciton migrations on the first excited-state manifold of delocalized exciton states. Nevertheless, charge transfers might contribute to the DNA spectroscopy and affect the present result if they effectively occur in a DNA helix. Also, our theory has not included quantum effects of contour ion and solvent, which could be important when charge transfers occur in a DNA helix. ${ }^{35}$

\section{ACKNOWLEDGMENTS}

This research is partially supported by Grant-in-Aids for Scientific Research from Japan Society for the Promotion of Science, Grant No. 17740278. M.C. is grateful for financial support from the CRI program of KOSEF (MOST, Korea). This research is also supported in part by the Global COE Program "International Center for Integrated Research and Advanced Education in Materials Science" (No. B-09) of the Ministry of Education, Culture, Sports, Science and Technology (MEXT) of Japan.

${ }^{1}$ R. G. Endres, D. L. Cox, and R. R. P. Singh, Rev. Mod. Phys. 76, 195 (2004).

${ }^{2}$ S. Marguet and D. Markovitsi, J. Am. Chem. Soc. 127, 5780 (2005).

${ }^{3}$ B. Bouvier, T. Gustavsson, D. Markovitsi, and P. Millié, Chem. Phys 275, 75 (2002).

${ }^{4}$ E. Emanuele, K. Zakrzewska, D. Markovitsi, R. Lavery, and P. Millié, J. Phys. Chem. B 109, 16109 (2005).

${ }^{5}$ A. Czader and E. R. Bittner, J. Chem. Phys. 128, 035101 (2008).

${ }^{6}$ B. Bouvier, J. P. Dognon, R. Lavery, D. Markovitsi, P. Millié, D. Onidas, and K. Zakrzewska, J. Phys. Chem. B 107, 13512 (2003).

${ }^{7}$ D. Onidas, D. Markovitsi, S. Marguet, A. Sharonov, and T. Gustavsson, J. Phys. Chem. B 106, 11367 (2002).

${ }^{8}$ P. M. Hare, C. E. Crespo-Hernández, and B. Kohler, Proc. Natl. Acad.
Sci. U.S.A. 104, 435 (2007)

${ }^{9}$ C. E. Crespo-Hernández, B. Cohen, and B. Kohler, Nature (London) 436, 1141 (2005).

${ }^{10}$ E. R. Bittner, J. Chem. Phys. 125, 094909 (2006).

${ }^{11}$ E. R. Bittner, J. Photochem. Photobiol., A 190, 328 (2007).

${ }^{12}$ E. Emanuele, D. Markovitsi, P. Millié, and K. Zakrzewska, ChemPhysChem 6, 1387 (2005).

${ }^{13}$ D. Markovitsi, A. Sharonov, D. Onidas, and T. Gustavsson, ChemPhysChem 4, 303 (2003).

${ }^{14}$ D. Markovitsi, D. Onidas, F. Talbot, S. Marguet, T. Gustavsson, and E. Lazzarotto, J. Photochem. Photobiol., A 183, 1 (2006).

${ }^{15}$ S. Marguet, D. Markovitsi, and F. Talbot, J. Phys. Chem. B 110, 11037 (2006).

${ }^{16}$ D. Markovitsi, D. Onidas, T. Gustavsson, F. Talbot, and E. Lazzarotto, J. Am. Chem. Soc. 127, 17130 (2005).

${ }^{17}$ I. Buchvarov, Q. Wang, M. Raytchev, A. Trifonov, and T. Fiebig, Proc. Natl. Acad. Sci. U.S.A. 104, 4794 (2007).

${ }^{18}$ C. Lee, K.-H. Park, and M. Cho, J. Chem. Phys. 125, 114508 (2006); C. Lee and M. Cho, ibid. 125, 114509 (2006); C. Lee, K.-H. Park, J.-A. Kim, S. Hahn, and M. Cho, ibid. 125, 114510 (2006); C. Lee and M. Cho, ibid. 126, 145102 (2007).

${ }^{19}$ H.-D. Kim, Y. Tanimura, and M. Cho, J. Chem. Phys. 127, 075101 (2007).

${ }^{20}$ G. R. Fleming and M. Cho, Annu. Rev. Phys. Chem. 47, 103 (1996).

${ }^{21}$ G. S. Engel, T. R. Calhoun, E. L. Read, T.-K. Ahn, T. Mančal, Y.-C. Cheng, R. E. Blankenship, and G. R. Fleming, Nature (London) 446, 782 (2007).

${ }^{22}$ M. Cho, H. M. Vaswani, T. Brixner, J. Stenger, and G. R. Fleming, J. Phys. Chem. B 109, 10542 (2005).

${ }^{23}$ M. Yang and G. R. Fleming, Chem. Phys. 282, 163 (2002).

${ }^{24}$ S. Mukamel, Principles of Nonlinear Optical Spectroscopy (Oxford University Press, New York, 1999).

${ }^{25}$ W. M. Zhang, T. Meier, V. Chernyak, and S. Mukamel, J. Chem. Phys. 108, 7763 (1998)

${ }^{26}$ This is because, although the derived time-evolution equation in Ref. 19 is actually correct, we recently found that the paper has a few mistakes in its derivation. We have shown the corrected derivation in this paper. The erratum paper has been published in J. Chem. Phys. 128, 129904 (2008).

${ }^{27}$ Y. Tanimura, J. Phys. Soc. Jpn. 75, 082001 (2006).

${ }^{28}$ M. Khalil, N. Demirdöven, and A. Tokmakoff, J. Chem. Phys. 121, 362 (2004).

${ }^{29}$ In Ref. 19, the last term in Eq. (50) was ignored. The erratum paper will be published in J. Chem. Phys. 128, 129904 (2008).

${ }^{30}$ R. Jimenez, F. van Mourik, J. Y. Yu, and G. R. Fleming, J. Phys. Chem. B 101, 7350 (1997).

${ }^{31}$ I. Barvík, V. Č́ápek, and P. Heřman, J. Lumin. 83-84, 105 (1999).

${ }^{32}$ V. Novoderezhkin, M. Wendling, and R. van Grondelle, J. Phys. Chem. B 107, 11534 (2003)

${ }^{33}$ G. C. Schatz and M. A. Ratner, Quantum Mechanics in Chemistry (Dover, New York, 2001).

${ }^{34}$ B. Giese, J. Amaudrut, A.-K. Köhler, M. Spormann, and S. Wessely, Nature (London) 412, 318 (2001).

${ }^{35}$ F. Santoro, V. Barone, T. Gustavsson, and R. Improta, J. Am. Chem. Soc. 128, 16312 (2006). 